\title{
PTF 10bzf (SN 2010ah): A BROAD-LINE Ic SUPERNOVA DISCOVERED BY THE PALOMAR TRANSIENT FACTORY
}

\author{
A. Corsi ${ }^{1}$, E. O. Ofer ${ }^{2,22}$, D. A. Frail ${ }^{3}$, D. Poznanski ${ }^{4,5,22}$, I. ArCavi ${ }^{6}$, A. Gal-Yam ${ }^{6}$, S. R. Kulkarni ${ }^{2}$, K. Hurley ${ }^{7}$, \\ P. A. Mazzali ${ }^{8,9}$, D. A. Howell ${ }^{10,11}$, M. M. Kasliwal 2 , Y. Green ${ }^{6}$, D. Murray ${ }^{10,11}$, M. Sullivan ${ }^{12}$, D. Xu ${ }^{6}$, S. Ben-Ami ${ }^{6}$, \\ J. S. Bloom ${ }^{5}$, S. B. Cenko ${ }^{5}$, N. M. LaW ${ }^{13}$, P. Nugent ${ }^{4,5}$, R. M. QuimbY ${ }^{2}$, V. Pal'shin ${ }^{14}$, J. Cummings ${ }^{15}$, V. Connaughton ${ }^{16}$,

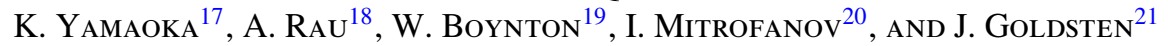 \\ ${ }^{1}$ LIGO Laboratory, California Institute of Technology, MS 100-36, Pasadena, CA 91125, USA; corsi@ caltech.edu \\ ${ }^{2}$ Cahill Center for Astrophysics, California Institute of Technology, Pasadena, CA 91125, USA \\ ${ }^{3}$ National Radio Astronomy Observatory, P.O. Box 0, Socorro, NM 87801, USA \\ ${ }^{4}$ Computational Cosmology Center, Lawrence Berkeley National Laboratory, 1 Cyclotron Road, Berkeley, CA 94720, USA \\ ${ }^{5}$ Department of Astronomy, 601 Campbell Hall, University of California, Berkeley, CA 94720-3411, USA \\ ${ }^{6}$ Department of Particle Physics and Astrophysics, The Weizmann Institute of Science, Rehovot 76100, Israel \\ ${ }^{7}$ Space Sciences Laboratory, University of California Berkeley, 7 Gauss Way, Berkeley, CA 94720, USA \\ ${ }^{8}$ INAF-Osservatorio Astronomico, vicolo dell'Osservatorio 5, I-35122 Padova, Italy \\ ${ }^{9}$ Max-Planck Institut für Astrophysik, Karl-Schwarzschildstr. 1, D-85748 Garching, Germany \\ ${ }^{10}$ Las Cumbres Observatory Global Telescope Network, Inc., Santa Barbara, CA 93117, USA \\ ${ }^{11}$ Department of Physics, University of California Santa Barbara, Santa Barbara, CA 93106, USA \\ ${ }^{12}$ Department of Physics (Astrophysics), University of Oxford, DWB, Keble Road, Oxford OX1 3RH, UK \\ ${ }^{13}$ Dunlap Institute for Astronomy and Astrophysics, University of Toronto, 50 St. George Street, Toronto M5S 3H4, Ontario, Canada \\ ${ }^{14}$ Ioffe Physico-Technical Institute of the Russian Academy of Sciences, St. Petersburg, Russian Federation \\ ${ }^{15}$ University of Maryland, Baltimore County (UMBC), 1000 Hilltop Circle, Baltimore, MD 21250, USA \\ ${ }^{16}$ Center for Space Plasma \& Aeronomic Research, University of Alabama in Huntsville, Huntsville, AL 35899, USA \\ ${ }^{17}$ Department of Physics and Mathematics, Aoyama Gakuin University, Kanagawa, Japan \\ ${ }^{18}$ Max-Planck-Institut für extraterrestrische Physik, D-85748 Garching, Germany
${ }^{19}$ Department of Planetary Sciences, University of Arizona, Tucson, AZ 85721, USA \\ ${ }^{20}$ Space Research Institute, Moscow, Russian Federation \\ ${ }^{21}$ Applied Physics Laboratory, Johns Hopkins University, Laurel, MD 20723, USA \\ Received 2011 January 21; accepted 2011 August 7; published 2011 October 19
}

\begin{abstract}
We present the discovery and follow-up observations of a broad-line Type Ic supernova (SN), PTF 10bzf (SN 2010ah), detected by the Palomar Transient Factory (PTF) on 2010 February 23 . The SN distance is $\cong 218 \mathrm{Mpc}$, greater than GRB 980425/SN 1998bw and GRB 060218/SN 2006aj, but smaller than the other SNe firmly associated with gamma-ray bursts (GRBs). We conducted a multi-wavelength follow-up campaign with Palomar 48 inch, Palomar 60 inch, Gemini-N, Keck, Wise, Swift, the Allen Telescope Array, Combined Array for Research in Millimeter-wave Astronomy, Westerbork Synthesis Radio Telescope, and Expanded Very Large Array. Here we compare the properties of PTF 10bzf with those of SN 1998bw and other broad-line SNe. The optical luminosity and spectral properties of PTF 10bzf suggest that this SN is intermediate, in kinetic energy and amount of ${ }^{56} \mathrm{Ni}$, between non-GRB-associated SNe like 2002ap or 1997ef, and GRB-associated SNe like 1998bw. No X-ray or radio counterpart to PTF 10bzf was detected. X-ray upper limits allow us to exclude the presence of an underlying X-ray afterglow as luminous as that of other SN-associated GRBs such as GRB 030329 or GRB 031203. Early-time radio upper limits do not show evidence for mildly relativistic ejecta. Late-time radio upper limits rule out the presence of an underlying off-axis GRB, with energy and wind density similar to the SN-associated GRB 030329 and GRB 031203. Finally, by performing a search for a GRB in the time window and at the position of PTF 10bzf, we find that no GRB in the interplanetary network catalog could be associated with this SN.
\end{abstract}

Key words: gamma-ray burst: general - radiation mechanisms: non-thermal - supernovae: general - supernovae: individual (PTF 10bzf)

Online-only material: color figures

\section{INTRODUCTION}

The Palomar Transient Factory ${ }^{23}$ (PTF; Law et al. 2009; Rau et al. 2009) is an ongoing project optimized for detecting optical transients in the local universe. One of its main objectives is the collection of a large sample of core-collapse supernovae (SNe; e.g., Arcavi et al. 2010), for which multicolor optical light curves and spectra can be obtained through dedicated follow-up resources.

The explosive death of a SN massive progenitor occurs when its iron core collapses to a neutron star or a black hole. Core-

\footnotetext{
${ }^{22}$ Einstein Fellow.

23 http://www.astro.caltech.edu//ptf/
}

collapse $\mathrm{SNe}$ are either of Type $\mathrm{Ib} / \mathrm{Ic}$ if the hydrogen envelope of the progenitor is lost, or else of Type II (Filippenko 1997). While the total kinetic energy released in the explosion is of the order of $10^{51} \mathrm{erg}$, roughly the same as the energy of the jet that makes a gamma-ray burst (GRB), core-collapse $\mathrm{SNe}$ are in general not accompanied by highly relativistic mass ejection and are visible from all angles.

The discovery of an association between a Type Ic SN and a long-duration GRB in 1998 (Galama et al. 1998; Kulkarni et al. 1998) strongly supported the collapsar scenario (e.g., MacFadyen \& Woosley 1999a; Mészáros 2006; Woosley \& Bloom 2006, and references therein). This was one of the most unusual Type Ic SNe seen up to that time: more luminous than typical Type Ic SNe, with broad lines and strong radio emission 
Table 1

Summary of Light-curve Properties

\begin{tabular}{|c|c|c|c|c|c|}
\hline $\mathrm{SN}$ & Type & $\begin{array}{c}\text { Associated } \\
\text { GRB }\end{array}$ & $\begin{array}{c}d_{\mathrm{L}} \\
(\mathrm{Mpc})\end{array}$ & $\begin{array}{c}M_{\mathrm{R}} \\
(\mathrm{mag})\end{array}$ & $M_{\mathrm{Ni}} / M_{\odot}$ \\
\hline SN 1998bw ${ }^{a}$ & Engine-driven BL-Ic & GRB 980425 & 37 & $-19.36 \pm 0.05$ & $0.4-0.5$ \\
\hline SN $2003 \mathrm{dh}^{\mathrm{b}}$ & Engine-driven BL-Ic & GRB 030329 & 810 & $\approx-19$ & $0.25-0.45$ \\
\hline SN 20031w ${ }^{c}$ & Engine-driven BL-Ic & GRB 031203 & 477 & $-19.90 \pm 0.08$ & $0.45-0.65$ \\
\hline SN 2006aj ${ }^{d}$ & Engine-driven BL-Ic & GRB 060218 & 140 & $-18.81 \pm 0.06$ & 0.21 \\
\hline SN 2010bh ${ }^{\mathrm{e}}$ & Engine-driven BL-Ic & GRB 100316D & 261 & $\ldots$ & $\ldots$ \\
\hline SN 2009bb ${ }^{f}$ & Engine-driven BL-Ic & None & 40 & $-18.56 \pm 0.28$ & $0.16-0.28$ \\
\hline SN 2003jdg & BL-Ic & None & 78 & $-18.94 \pm 0.30$ & $0.26-0.45$ \\
\hline SN 2002ap ${ }^{\mathrm{h}}$ & BL-Ic & None & 7.8 & $-17.50 \pm 0.32$ & $0.06-0.12$ \\
\hline
\end{tabular}

Notes. See also Woosley \& Bloom (2006, and references therein) for a recent review.

a Galama et al. (1998), Iwamoto et al. (1998), and Nakamura et al. (2001).

${ }^{b}$ Hjorth et al. (2003), Matheson et al. (2003), and Deng et al. (2005).

c Malesani et al. (2004) and Mazzali et al. (2006b).

d Mazzali et al. (2006a), Pian et al. (2006), Soderberg et al. (2006a), and Valenti et al. (2008).

e Starling et al. (2010).

${ }^{\mathrm{f}}$ Pignata et al. (2011).

g Mazzali et al. (2005), Valenti et al. (2008), and Drout et al. (2010)

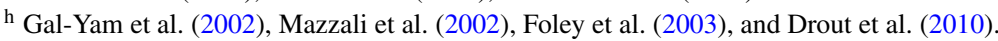

indicating a relativistic expansion speed $(\Gamma \sim 2$; Kulkarni et al. 1998).

Since 1998, a total of five associations between GRBs and $\mathrm{SNe}$ have been spectroscopically confirmed (Table 1). GRBs with spectroscopically confirmed SNe are generally underluminous and sub-energetic in comparison to typical long GRBs. A notable exception, though, is GRB 030329 associated with SN 2003dh that represents the first solid evidence for a connection between ordinary GRBs and SNe (Garnavich et al. 2003; Hjorth et al. 2003; Kawabata et al. 2003; Matheson et al. 2003; Stanek et al. 2003).

After GRB 030329, an SN-like brightening was reported at the position of GRB 031203, and SN 2003lw was then photometrically (Bersier et al. 2004; Cobb et al. 2004; Malesani et al. 2004; Gal-Yam et al. 2004) and spectroscopically (Malesani et al. 2004) confirmed to be associated with this GRB. Broad spectral features similar to SN 1998bw also characterized SN 20031w.

The understanding of the GRB-SN connection has further progressed thanks to the discovery of GRB/XRF 060218 (Pian et al. 2006; Modjaz et al. 2006; Sollerman et al. 2006; Ferrero et al. 2006), associated with SN 2006aj. The prompt spectrum of this GRB showed evidence for a typical non-thermal component (as observed also in ordinary GRBs) plus a thermal component. This last suggested that the shock breakout of an associated SN was for the first time being observed (Campana et al. 2006; Waxman et al. 2007). A different central engine (a magnetar rather than a black hole) was also suggested by several authors to explain the long duration and lower luminosity of GRB 060218 (Mazzali et al. 2006b; Soderberg et al. 2006a; Toma et al. 2007).

The breakout of a shock through the stellar surface is predicted to be the first electromagnetic signal marking the birth of a SN. The typical frequency of the emission is in the soft $\gamma$-rays for core-collapse SNe (Grassberg et al. 1971; Chevalier 1976, 1992; Waxman et al. 2007; Chevalier \& Fransson 2008; Katz et al. 2010; Nakar \& Sari 2010; Balberg \& Loeb 2011; Katz et al. 2011; Nagakura et al. 2011; Rabinak \& Waxman 2011). Since XRF 060218, several shock breakout candidate events have been proposed (Soderberg et al. 2008; Gezari et al. 2008; Schawinski et al. 2008; Modjaz et al. 2009; Ofek et al. 2010b). Multi-wavelength observations of core-collapse SNe are fundamental to probe the breakout phase.

The most recent spectroscopically confirmed association between a GRB and a $\mathrm{SN}$ is represented by the case of GRB 100316D (Starling et al. 2010) and SN 2010bh (Bufano et al. 2010; Chornock et al. 2010; Wiersema et al. 2010; Cano et al. 2011). GRB 100316D was a long-duration, soft-spectrum GRB, resembling GRB 060218 in these properties.

While the above-mentioned observations have clearly established a connection between long-duration GRBs and Type Ic core-collapse $\mathrm{SNe}$, however, what makes some broad-line Type Ic SNe have an accompanying GRB is still a mystery. Some long GRBs are clearly not associated with a SN, e.g., GRB 060505 (Ofek et al. 2007) and GRB 060614 (Della Valle et al. 2006; Fynbo et al. 2006; Gal-Yam et al. 2006a). On the other hand, SN 1997ef remains so far the most energetic peculiar Type Ic SN without a clear GRB association (e.g., Mazzali et al. 2000), and the broad-line Type Ic SN 2009bb (Soderberg et al. 2010) showed clear evidence of very high expansion velocity (as normally seen in GRB-related SNe), but no clear GRB association. However, it is remarkable that all GRB-related SNe discovered to date are broad-line Type Ic SNe.

Theoretically, in order to produce a relativistic jet from a collapsing star to power the observed GRB, the stellar core has to carry a high angular momentum (Woosley \& Heger 2006), so that the spin axis provides a natural preferred propagation direction for the jet. Moreover, the jet needs to be "clean," with small baryon contamination, so that it can achieve a relativistic speed, with Lorentz factor $\Gamma$ typically greater than 100 (Piran 1999; Lithwick \& Sari 2001; Liang et al. 2010; Abdo et al. 2009b, 2009a, 2009c; Corsi et al. 2010). The outflow also needs to be collimated, with an aperture angle of the order of $1^{\circ}-10^{\circ}$ for bright GRBs (Frail et al. 2001; Bloom et al. 2003; Liang et al. 2008; Racusin et al. 2009).

Several types of GRB central engine have been discussed, the leading candidate being a black hole plus torus system (Woosley 1993; MacFadyen \& Woosley 1999b; Proga \& Begelman 2003; Zhang et al. 2003). An alternative candidate is a rapidly spinning, highly magnetized neutron star (magnetar; e.g., Usov 1992; Thompson et al. 2004; Bucciantini et al. 2008; Metzger 
et al. 2011). In the black hole scenario, the first energy source is the accretion power from the torus. On the other hand, the main power of a millisecond magnetar engine is its spin-down power.

Whatever the nature of the GRB central engine is, it has to generate both a narrowly collimated, highly relativistic jet to make the GRB and a wide-angle, sub-relativistic outflow responsible for exploding the star and making the SN. To some extent, the two components may vary independently, so it is possible to produce a variety of jet energies and SN luminosities. Woosley \& Zhang (2007) have shown that at least $\mathrm{a} \sim 10^{48} \mathrm{erg} \mathrm{s}^{-1}$ power is required for a jet to escape a massive star before that star either explodes or is accreted, and lower energy and "suffocated" bursts may be particularly prevalent when the metallicity is high, i.e., in the modern universe at low redshift.

A key prediction of jet models for GRBs (e.g., Piran 2004), combined with the association of long GRBs with Type Ib/Ic core-collapse SNe (e.g., Woosley \& Bloom 2006), is that some (spherical) SN explosions will be accompanied by off-axis GRBs, whose gamma-ray signal is missed because the jet is not pointed at us, but whose afterglow emission could be visible at lower energies (from radio to X-rays), once the jet decelerates. These are also referred to as "orphan afterglows." Detecting an off-axis GRB would constitute a direct proof of the popular jet model for GRBs but, after a decade of efforts (Berger et al. 2003b; Gal-Yam et al. 2006b; Soderberg et al. 2006a), such a detection has not yet been achieved. Orphan afterglows may also be produced by "dirty fireballs," i.e., cosmological fireballs whose ejecta carry too many baryons to produce a GRB. Multicolor, wide-area searches, combined with radio follow-up, can help to distinguish between off-axis GRBs and dirty fireballs (Perna \& Loeb 1998; Rhoads 2003).

Based on radio follow-up campaigns of Type Ib/c SNe (Berger et al. 2003b; Soderberg et al. 2010), it is now believed that no more than $1 \%$ of $\mathrm{SNe}$ Type $\mathrm{Ib} / \mathrm{c}$ are powered by central engines. These observational facts are giving us hints at the fundamental role that broad-line Type Ic SNe play in enginedriven explosions. GRBs are known to prefer low-metallicity dwarf galaxies (e.g., Fynbo et al. 2003; Fruchter et al. 2006), and to be associated only with a particular subclass of core-collapse SNe: broad-line Ic events. Previous searches, however, targeted mostly regular $\mathrm{SNe} \mathrm{Ib} / \mathrm{Ic}$, residing in giant, high-metallicity hosts. This is why broad-line Ic events deserve to be studied with particular attention (Modjaz et al. 2008).

Multi-wavelength follow-up campaigns of broad-line $\mathrm{Ib} / \mathrm{c}$ $\mathrm{SNe}$ are especially important to probe high expansion velocities and/or any associated GRB. Moreover, because of their low (electromagnetic) luminosity compared to GRBs, usually $\mathrm{SNe}$ are observed at non-cosmological distances $(z \lesssim 1)$. Thus, broad-line $\mathrm{Ib} / \mathrm{c} \mathrm{SNe}$ also represent a tool to search for nearby GRB explosions and understand their connection with cosmological GRBs (e.g., Norris 2002; Podsiadlowski et al. 2004; Guetta \& Della Valle 2007; Liang et al. 2007; Virgili et al. 2009).

In this paper, we present the discovery of a broadline Type Ic SN, PTF 10bzf (SN 2010ah), detected by PTF. This event is interesting for two reasons. First, as a broad-line SN located at a distance smaller than most GRB-associated events (except for GRB 980425/SN 1998bw and GRB 060218/SN 2006aj, see Table 1). Next, this event enjoyed a rich radio-to-X-ray follow-up campaign. In what follows, we first describe the observations that led to the discovery of PTF 10bzf (Section 2), and its multi-wavelength follow-up campaign (Section 3). Then, we compare PTF 10bzf with SN 1998bw and other GRB-associated SNe, and describe the results of an associated GRB search (Section 4). Finally, we conclude in Section 5.

\section{OBSERVATIONS AND DATA REDUCTION}

On 2010 February 23.5038 (hereafter all times are given in UTC), we discovered a broad-line Type-Ic SN, PTF 10bzf, visible at a magnitude of $R \approx 18.86$ (see Table 2 and Figure 1), in a $60 \mathrm{~s}$ exposure image taken with the Palomar 48 inch telescope (P48). The SN was not seen in previous images of the same field taken on 2010 February 19.4392, down to a limiting magnitude of $R>21.3$. The SN J2000 position is R.A. = 11:44:02.99, decl. $=+55: 41: 27.6$ (Ofek et al. 2010a), $\cong 5^{\prime \prime} \cdot 2$ offset, and at a position angle of $\cong 5 \mathrm{deg}$ (north through east) about the position of the galaxy $\operatorname{SDSS}^{24} \mathrm{~J} 114402.98+554122.5$.

Digital copies of our data can be downloaded directly from the Weizmann Institute of Science Experimental Astrophysics Spectroscopy System (WISEASS ${ }^{25}$; S. E. Yaron et al. 2011, in preparation).

\subsection{Optical Photometry}

P48 observations of the PTF 10bzf field were performed with the Mould- $R$ filter. A high-quality image produced by stacking several images of the same field (obtained between 2009 May and 2009 June) was used as a reference and subtracted from the individual images. Photometry was performed relative to the $r$-band magnitudes of 10 SDSS reference stars in the field, including $r-i$ color term corrections, using an aperture of 2 arcsec radius, and applying aperture corrections to account for systematic errors and errors introduced by the subtraction process. Aperture corrections are all below $\approx 0.04$ mag. All the P48 photometry is listed in Table 2. The P48 calibrated light curve of PTF 10bzf is plotted in Figure 2.

P60 observations were carried out in the $B, g, r, i, z$ bands. We also observed the PTF 10bzf field with the $1 \mathrm{~m}$ telescope at the Wise observatory ${ }^{26}$ using $B V R I$ filters (see Table 2). For both P60 and Wise observations, image subtraction was performed using the common point-spread function method via the "mkdifflc" routine (Gal-Yam et al. 2004, 2008). Errors on the P60 and Wise data are estimated by using "artificial" sources at a brightness similar to that of the real $\mathrm{SN}$, with the scatter in their magnitudes providing an estimate of the error due to subtraction residuals. The measured magnitude was calibrated against the magnitudes of SDSS stars in the same field (see Figure 1). The calibration procedure described in Jordi et al. (2006) was used for the $B V R I$ data. Calibration errors were summed in quadrature with the subtraction errors.

\subsection{Spectroscopy}

Gemini North GMOS ${ }^{27}$ (Hook et al. 2004) spectra were taken on 2010 March 2 (Program ID: GN-2010A-Q-20), using a 1" slit, with the B600 and R400 gratings. The observations had an

\footnotetext{
24 Sloan Digital Sky Survey (York et al. 2000).

25 http://www.weizmann.ac.il/astrophysics/wiseass/

26 http://wise-obs.tau.ac.il/

27 The Gemini Observatory is operated by the Association of Universities for Research in Astronomy, Inc., under a cooperative agreement with the NSF on behalf of the Gemini partnership: the National Science Foundation (United States), the Science and Technology Facilities Council (United Kingdom), the National Research Council (Canada), CONICYT (Chile), the Australian Research Council (Australia), Ministério da Ciência e Tecnologia (Brazil), and Ministerio de Ciencia, Tecnología e Innovación Productiva (Argentina).
} 
Table 2

PTF 10bzf Follow-up Campaign

\begin{tabular}{|c|c|c|c|c|c|}
\hline $\begin{array}{l}\text { JD-2455251.004 } \\
\text { (days since Feb 23.504) }\end{array}$ & Telescope & $\begin{array}{l}\Delta t \\
(\mathrm{~s})\end{array}$ & Band & Mag or Flux & Reference \\
\hline-4.061 & $\mathrm{P} 48$ & 60 & Mould- $R$ & $>21.3$ & ATEL 2470 \\
\hline 0.000 & P48 & 60 & Mould- $R$ & $18.863 \pm 0.041$ & ATEL 2470 \\
\hline 0.045 & $\mathrm{P} 48$ & 60 & Mould- $R$ & $18.850 \pm 0.056$ & This paper \\
\hline 18.038 & P48 & 60 & Mould- $R$ & $18.362 \pm 0.040$ & This paper \\
\hline 18.921 & P48 & 60 & Mould- $R$ & $18.527 \pm 0.030$ & This paper \\
\hline 18.966 & P48 & 60 & Mould- $R$ & $18.495 \pm 0.030$ & This paper \\
\hline 21.832 & $\mathrm{P} 48$ & 60 & Mould- $R$ & $18.599 \pm 0.036$ & This paper \\
\hline 21.876 & P48 & 60 & Mould- $R$ & $18.618 \pm 0.039$ & This paper \\
\hline 24.866 & $\mathrm{P} 48$ & 60 & Mould- $R$ & $18.791 \pm 0.033$ & This paper \\
\hline 24.911 & P48 & 60 & Mould- $R$ & $18.735 \pm 0.034$ & This paper \\
\hline 28.727 & $\mathrm{P} 48$ & 60 & Mould- $R$ & $18.966 \pm 0.062$ & This paper \\
\hline 28.771 & $\mathrm{P} 48$ & 60 & Mould- $R$ & $18.861 \pm 0.042$ & This paper \\
\hline 31.636 & $\mathrm{P} 48$ & 60 & Mould- $R$ & $19.12 \pm 0.11$ & This paper \\
\hline 31.679 & $\mathrm{P} 48$ & 60 & Mould- $R$ & $19.061 \pm 0.085$ & This paper \\
\hline 33.650 & $\mathrm{P} 48$ & 60 & Mould- $R$ & $19.18 \pm 0.11$ & This paper \\
\hline 33.693 & $\mathrm{P} 48$ & 60 & Mould- $R$ & $19.093 \pm 0.081$ & This paper \\
\hline 38.758 & $\mathrm{P} 48$ & 60 & Mould- $R$ & $19.381 \pm 0.050$ & This paper \\
\hline 38.802 & $\mathrm{P} 48$ & 60 & Mould- $R$ & $19.359 \pm 0.053$ & This paper \\
\hline 39.857 & P48 & 60 & Mould- $R$ & $19.393 \pm 0.058$ & This paper \\
\hline 39.901 & $\mathrm{P} 48$ & 60 & Mould- $R$ & $19.401 \pm 0.057$ & This paper \\
\hline 42.670 & $\mathrm{P} 48$ & 60 & Mould- $R$ & $19.547 \pm 0.062$ & This paper \\
\hline 42.713 & $\mathrm{P} 48$ & 60 & Mould- $R$ & $19.480 \pm 0.065$ & This paper \\
\hline 43.829 & $\mathrm{P} 48$ & 60 & Mould- $R$ & $19.576 \pm 0.061$ & This paper \\
\hline 43.873 & $\mathrm{P} 48$ & 60 & Mould- $R$ & $19.723 \pm 0.065$ & This paper \\
\hline 44.926 & $\mathrm{P} 48$ & 60 & Mould- $R$ & $19.597 \pm 0.053$ & This paper \\
\hline 44.962 & $\mathrm{P} 48$ & 60 & Mould- $R$ & $19.531 \pm 0.059$ & This paper \\
\hline 45.983 & $\mathrm{P} 48$ & 60 & Mould- $R$ & $19.696 \pm 0.067$ & This paper \\
\hline 45.984 & P48 & 60 & Mould- $R$ & $19.764 \pm 0.075$ & This paper \\
\hline 60.737 & P48 & 60 & Mould- $R$ & $19.800 \pm 0.093$ & This paper \\
\hline 60.781 & $\mathrm{P} 48$ & 60 & Mould- $R$ & $19.89 \pm 0.11$ & This paper \\
\hline 61.783 & P48 & 60 & Mould- $R$ & $19.96 \pm 0.12$ & This paper \\
\hline 61.826 & $\mathrm{P} 48$ & 60 & Mould- $R$ & $20.23 \pm 0.13$ & This paper \\
\hline 62.835 & $\mathrm{P} 48$ & 60 & Mould- $R$ & $20.27 \pm 0.19$ & This paper \\
\hline 62.878 & $\mathrm{P} 48$ & 60 & Mould- $R$ & $20.15 \pm 0.17$ & This paper \\
\hline 65.782 & $\mathrm{P} 48$ & 60 & Mould- $R$ & $20.02 \pm 0.11$ & This paper \\
\hline 65.857 & $\mathrm{P} 48$ & 60 & Mould- $R$ & $19.91 \pm 0.13$ & This paper \\
\hline 66.888 & $\mathrm{P} 48$ & 60 & Mould- $R$ & $20.06 \pm 0.11$ & This paper \\
\hline 66.932 & $\mathrm{P} 48$ & 60 & Mould- $R$ & $20.10 \pm 0.15$ & This paper \\
\hline 68.649 & $\mathrm{P} 48$ & 60 & Mould- $R$ & $20.173 \pm 0.093$ & This paper \\
\hline 68.692 & P48 & 60 & Mould- $R$ & $20.198 \pm 0.098$ & This paper \\
\hline 69.771 & $\mathrm{P} 48$ & 60 & Mould- $R$ & $20.299 \pm 0.087$ & This paper \\
\hline 69.815 & $\mathrm{P} 48$ & 60 & Mould- $R$ & $20.159 \pm 0.079$ & This paper \\
\hline 43.840 & $\mathrm{P} 60$ & 180 & $r$ & $19.47 \pm 0.13$ & This paper \\
\hline 43.841 & P60 & 180 & $i$ & $19.51 \pm 0.32$ & This paper \\
\hline 44.898 & P60 & 180 & $z$ & $19.86 \pm 0.57$ & This paper \\
\hline 44.901 & $\mathrm{P} 60$ & 180 & $B$ & $21.63 \pm 0.75^{\mathrm{a}}$ & This paper \\
\hline 49.960 & P60 & 180 & $g$ & $21.17 \pm 0.17$ & This paper \\
\hline 49.962 & $\mathrm{P} 60$ & 180 & $r$ & $19.587 \pm 0.070$ & This paper \\
\hline 49.964 & P60 & 180 & $i$ & $19.746 \pm 0.044$ & This paper \\
\hline 49.965 & P60 & 180 & $z$ & $18.99 \pm 0.31$ & This paper \\
\hline 49.967 & P60 & 180 & $B$ & $21.7 \pm 1.0^{\mathrm{a}}$ & This paper \\
\hline 53.993 & P60 & 180 & $r$ & $19.72 \pm 0.17$ & This paper \\
\hline 59.895 & P60 & 180 & $r$ & $19.77 \pm 0.20$ & This paper \\
\hline 65.904 & P60 & 180 & $i$ & $19.96 \pm 0.17$ & This paper \\
\hline 73.842 & P60 & 180 & $i$ & $20.15 \pm 0.17$ & This paper \\
\hline 73.844 & P60 & 180 & $r$ & $20.27 \pm 0.17$ & This paper \\
\hline 78.790 & P60 & 180 & $r$ & $20.38 \pm 0.16$ & This paper \\
\hline 79.790 & P60 & 180 & $i$ & $20.50 \pm 0.17$ & This paper \\
\hline 79.794 & P60 & 180 & $B$ & $21.82 \pm 0.83^{\mathrm{a}}$ & This paper \\
\hline 79.797 & $\mathrm{P} 60$ & 180 & $g$ & $20.88 \pm 0.16$ & This paper \\
\hline 80.771 & P60 & 180 & $z$ & $19.47 \pm 0.45$ & This paper \\
\hline 81.848 & P60 & 180 & $r$ & $20.207 \pm 0.084$ & This paper \\
\hline 99.798 & P60 & 180 & $i$ & $20.99 \pm 0.36$ & This paper \\
\hline 99.801 & P60 & 180 & $B$ & $22.5 \pm 1.3^{\mathrm{a}}$ & This paper \\
\hline
\end{tabular}


Table 2

(Continued)

\begin{tabular}{|c|c|c|c|c|c|}
\hline $\begin{array}{l}\text { JD-2455251.004 } \\
\text { (days since Feb 23.504) }\end{array}$ & Telescope & $\begin{array}{l}\Delta t \\
(\mathrm{~s})\end{array}$ & Band & Mag or Flux & Reference \\
\hline 99.803 & P60 & 180 & $g$ & $21.27 \pm 0.19$ & This paper \\
\hline 102.774 & P60 & 180 & $r$ & $20.72 \pm 0.12$ & This paper \\
\hline 102.776 & P60 & 180 & $B$ & $22.45 \pm 0.81^{\mathrm{a}}$ & This paper \\
\hline 102.780 & $\mathrm{P} 60$ & 180 & $g$ & $21.66 \pm 0.31$ & This paper \\
\hline 103.791 & P60 & 180 & $i$ & $20.80 \pm 0.27$ & This paper \\
\hline 24 & Wise (PI) & 600 & $B$ & $20.34 \pm 0.57^{b}$ & This paper \\
\hline 24 & Wise (PI) & 600 & V & $19.08 \pm 0.18^{\mathrm{b}}$ & This paper \\
\hline 24 & Wise (PI) & 600 & $R$ & $18.55 \pm 0.11^{b}$ & This paper \\
\hline 28 & Wise (PI) & 600 & $I$ & $18.42 \pm 0.20^{\mathrm{b}}$ & This paper \\
\hline 28 & Wise (PI) & 600 & $B$ & $20.60 \pm 0.64^{b}$ & This paper \\
\hline 28 & Wise (PI) & 600 & V & $19.36 \pm 0.27^{b}$ & This paper \\
\hline 28 & Wise (PI) & 600 & $R$ & $18.79 \pm 0.20^{\mathrm{b}}$ & This paper \\
\hline 28 & Wise (PI) & 600 & $I$ & $18.60 \pm 0.13^{\mathrm{b}}$ & This paper \\
\hline 35 & Wise (LAIWO) & 720 & V & $20.27 \pm 0.42^{b}$ & This paper \\
\hline 35 & Wise (LAIWO) & 720 & $R$ & $18.87 \pm 0.14^{b}$ & This paper \\
\hline 35 & Wise (LAIWO) & 720 & $I$ & $19.00 \pm 0.61^{\mathrm{b}}$ & This paper \\
\hline 38 & Wise (LAIWO) & 720 & $V$ & $19.87 \pm 0.69^{b}$ & This paper \\
\hline 38 & Wise (LAIWO) & 720 & $R$ & $19.57 \pm 0.34^{\mathrm{b}}$ & This paper \\
\hline 7 & Gemini & 450 & $g^{\mathrm{c}}$ & $18.6 \pm 0.3$ & $\overline{\text { ATEL } 2470}$ \\
\hline 7 & Gemini & 450 & $r^{\mathrm{c}}$ & $18.3 \pm 0.3$ & ATEL 2470 \\
\hline 7 & Gemini & 450 & $i^{\mathrm{c}}$ & $18.7 \pm 0.3$ & ATEL 2470 \\
\hline 12 & Keck & 240 & $g^{\mathrm{c}}$ & $18.91 \pm 0.3$ & This paper \\
\hline 12 & Keck & $2 \times 80$ & $r^{\mathrm{c}}$ & $18.48 \pm 0.3$ & This paper \\
\hline 12 & Keck & $2 \times 80$ & $i^{\mathrm{c}}$ & $18.70 \pm 0.3$ & This paper \\
\hline 8.52 & UVOT & $5 \times 10^{3}$ & $B$ & $18.73 \pm 0.10$ & ATEL 2471 \\
\hline 8.52 & UVOT & $5 \times 10^{3}$ & $U$ & $18.88 \pm 0.12$ & ATEL 2471 \\
\hline 8.52 & UVOT & $5 \times 10^{3}$ & $U V W 1$ & $20.07 \pm 0.18$ & ATEL 2471 \\
\hline 8.52 & UVOT & $5 \times 10^{3}$ & $U V W 2$ & $20.18 \pm 0.26$ & ATEL 2471 \\
\hline 12.80 & UVOT & $2.5 \times 10^{3}$ & $U$ & $19.68 \pm 0.14$ & ATEL 2471 \\
\hline 12.80 & UVOT & $2.5 \times 10^{3}$ & $U V W 1$ & $20.12 \pm 0.24$ & ATEL 2471 \\
\hline 8.52 & XRT & $5 \times 10^{3}$ & $0.3-10 \mathrm{keV}$ & $<1.3 \times 10^{-14} \mathrm{erg} \mathrm{s}^{-1} \mathrm{~cm}^{-2}$ & ATEL 2471 \\
\hline 12.80 & XRT & $2.5 \times 10^{3}$ & $0.3-10 \mathrm{keV}$ & $<2.7 \times 10^{-14} \mathrm{erg} \mathrm{s}^{-1} \mathrm{~cm}^{-2}$ & ATEL 2471 \\
\hline 9.69 & CARMA & $19.8 \times 10^{3}$ & $95 \mathrm{GHz}$ & $(-3.7 \pm 1.8) \times 10^{3} \mu \mathrm{Jy}$ & ATEL 2473 \\
\hline 10.14 & Allen & $4.8 \times 10^{3}$ & $3.09 \mathrm{GHz}$ & $<1.5 \times 10^{3} \mu \mathrm{Jy}$ & ATEL 2472 \\
\hline 17.69 & EVLA & $5.76 \times 10^{3}$ & $4.96 \mathrm{GHz}$ & $<33 \mu \mathrm{Jy}$ & ATEL 2483 \\
\hline 86.71 & EVLA & 3600 & $6 \mathrm{GHz}$ & $<36 \mu \mathrm{Jy}$ & This paper \\
\hline 276.9 & EVLA & 7200 & $4.96 \mathrm{GHz}$ & $<35 \mu \mathrm{Jy}$ & This paper \\
\hline 18.24 & WSRT & $28.8 \times 10^{3}$ & $4.8 \mathrm{GHz}$ & $<126 \mu \mathrm{Jy}$ & ATEL 2479 \\
\hline
\end{tabular}

Notes. Magnitudes are not corrected for Galactic extinction $(E(B-V)=0.012 \mathrm{mag}$; Schlegel et al. 1998). P48 and P60 observations are calibrated to SDSS (which is estimated to be on the $\mathrm{AB}$ system within \pm 0.01 mag in the $r, i$, and $g$ bands; within \pm 0.02 mag in the $z$ band). P48 errors include (in quadrature) zero-point calibration errors ( $\lesssim 0.01 \mathrm{mag})$, color term errors ( $\lesssim 0.03 \mathrm{mag})$, and aperture correction errors $(\lesssim 0.04 \mathrm{mag})$. For P48 detections, positive counts were collected in the subtracted images at the SN position and magnitudes are above the image $3 \sigma$ limiting magnitude. All upper limits are at $3 \sigma$.

${ }^{a} B$ magnitudes (in the Vega system) calibrated to SDSS using the conversions described in Jordi et al. (2006).

b $B V R I$ magnitudes (in the Vega system) calibrated to SDSS using the conversions described in Jordi et al. (2006).

${ }^{c}$ Synthetic photometry is referred to SDSS $g, r, i$ filters (in the AB system). Errors are dominated by flux calibration errors.

exposure time of $450 \mathrm{~s}$ (see Table 2), the airmass was 1.236, the sky position angle was $191^{\circ}$, and the standard star was Feige34. Standard data reduction was performed with IRAF V2.14, using the Gemini 1.10 reduction packages. The Gemini spectrum of PTF 10bzf is shown in Figure 3. A redshift of $z=0.0498 \pm 0.0003$ was derived from the host galaxy emission lines of $\mathrm{O}$ III, $\mathrm{H} \alpha, \mathrm{H} \beta, \mathrm{N}$ II, and $\mathrm{S}$ II. The spectrum (black line in Figure 3) resembles that of SN 1998bw at a similar epoch (red line in Figure 3 and Galama et al. 1998) and shows very broad lines, leading us to classify this SN as a broad-line Ic.
PTF 10bzf was also observed by Keck I/LRIS ${ }^{28}$ using a $1^{\prime \prime}$ slit, with the $400 / 8500$ grating plus $7847 \AA$ central wavelength on the red side and with the $400 / 3400$ grism on the blue side. The exposure time was $2 \times 80 \mathrm{~s}$ on the red side and $1 \times 240 \mathrm{~s}$ on the blue side (see Table 2). Keck data were reduced using the

\footnotetext{
28 The W. M. Keck Observatory is operated as a scientific partnership among the California Institute of Technology, the University of California and the National Aeronautics and Space Administration. The Observatory was made
} possible by the generous financial support of the W. M. Keck Foundation. 


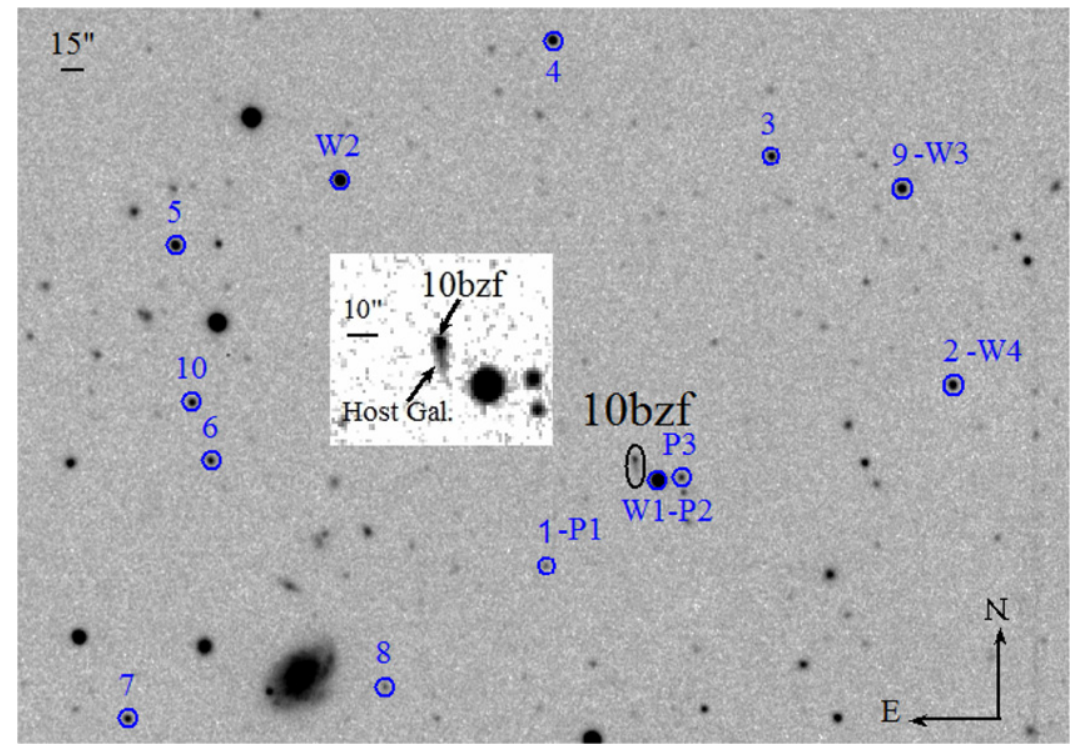

Figure 1. P48 discovery image of PTF $10 \mathrm{bzf}$ in the $R$ band. For clarity purposes, a circle of $5^{\prime \prime}$ radius marks the position of the 10 reference stars used for P48 photometry calibration (1-10), and of the four reference stars used for calibration of the Wise data (W1-W4). The stars labeled as P1-P3 were used for the calibration of P60 photometry. The position of PTF 10bzf and its host galaxy are enclosed by a black ellipse. The inset shows in more detail PTF 10bzf inside its host. The SN is located at R.A. $=11: 44: 02.99$ and decl. $=+55: 41: 27.6(\mathrm{~J} 2000)$.

(A color version of this figure is available in the online journal.)

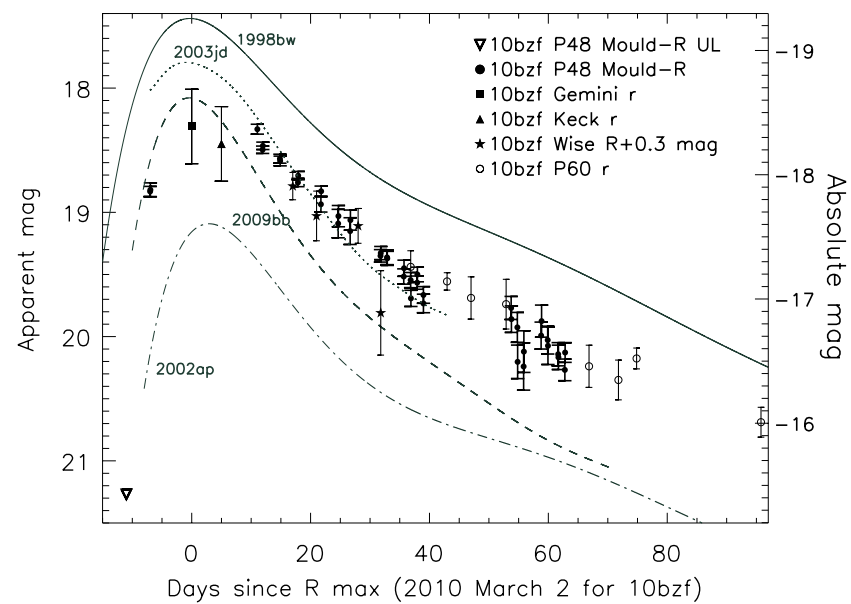

Figure 2. Light curve of PTF 10bzf corrected for Galactic extinction. Absolute magnitudes are plotted on the right axis. $\mathrm{P} 48$ data in Mould- $R$ filter (filled circles) and P60 data in $r$-band (open circles) are calibrated using $r$-band magnitudes of SDSS stars, and expressed in the AB system. For P48, we also include color term corrections estimated considering the $r-i$ SDSS colors of the reference stars. Synthetic photometry obtained from Gemini (filled square) and Keck (filled triangle) spectra is referred to SDSS $r$-band and expressed in the AB system. From the Gemini spectrum, we estimate $R-r \approx-0.1$ mag for the conversion from SDSS $r$-band magnitudes in the $\mathrm{AB}$ system to $R$-band magnitudes in the Vega system. Wise data in the $R$ filter and Vega system (stars) are calibrated to SDSS as described in Jordi et al. (2006). For comparison with P48 photometry, Wise data are shifted to account for $r-R \approx 0.3 \mathrm{mag}$ for the conversion from SDSS $r$-band magnitudes in the $\mathrm{AB}$ system, to Wise $R$-band magnitudes in the Vega system (Jordi et al. 2006). We also plot the $R$-band light-curve template of SN 1998bw (solid line), SN 2003jd (dotted line), SN 2009bb (dashed line), and SN 2002ap (dash-dotted line), rescaled to the redshift of PTF 10bzf. These templates are obtained by interpolating data retrieved from Galama et al. (1998) for SN 1998bw, Gal-Yam et al. (2002), Foley et al. (2003) for SN 2002ap, Valenti et al. (2008) for SN 2003jd, and Pignata et al. (2011) for SN 2009bb.

standard long-slit reduction packages developed in the IRAF environment (Figure 4).

Both Gemini and Keck spectra were used to derive synthetic photometry in SDSS $r$-, $g_{-}$, and $i$-bands (Table 2). Synthetic

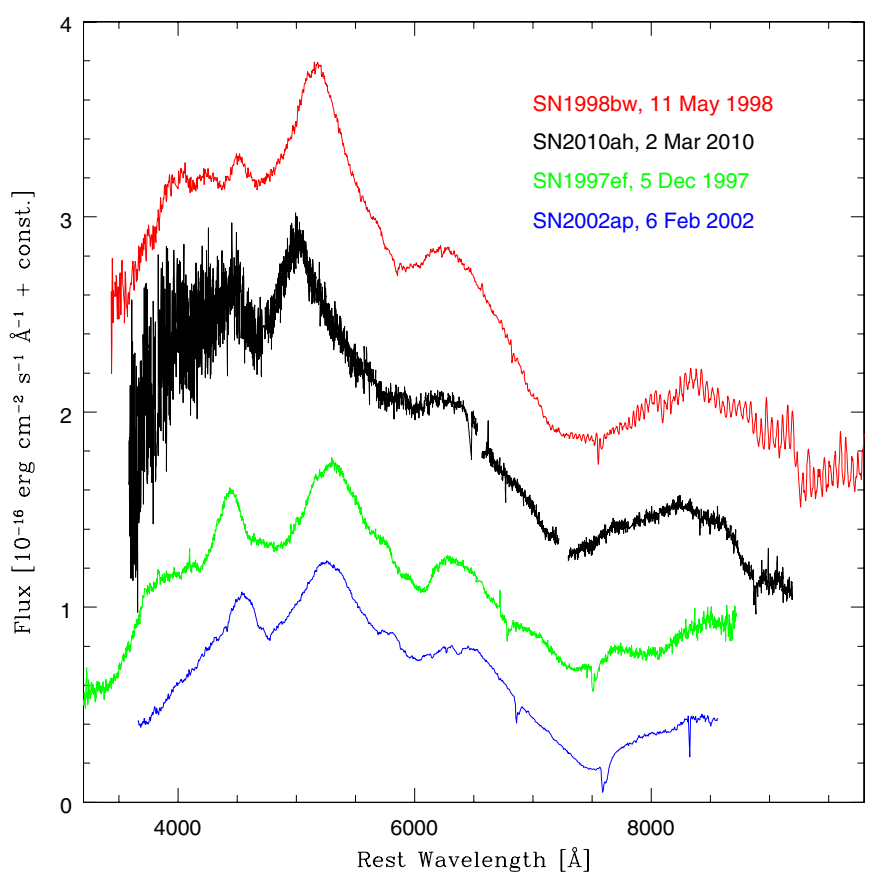

Figure 3. Gemini (black) spectrum of PTF 10bzf obtained on 2010 March 2, around the time of the $R$-band maximum. The spectrum is compared with that of SN 1997ef (green), SN 1998bw (red), and SN 2002ap (blue) at similar epochs. The SNe are ordered by peak luminosity. This ordering reveals a sequence also in line velocity and blending. The spectrum of SN $1998 \mathrm{bw}$ is the most affected by line broadening, as seen, for example, in the almost complete absence of the re-emission peak near $4500 \AA$ and by the complete blending of the O I 7773 and the Ca II IR triplet. PTF 10bzf is intermediate between SN 1998bw and the two non-GRB SNe 1997ef and 2002ap in both of these respects. Spectral data were retrieved from Mazzali et al. (2000) for SN 19997ef, Patat et al. (2001) for SN 1998bw, and Mazzali et al. (2002) for SN 2002ap. Main telluric lines have been removed from the PTF 10bzf spectrum.

(A color version of this figure is available in the online journal.)

photometry was performed as described in Poznanski et al. (2002). 


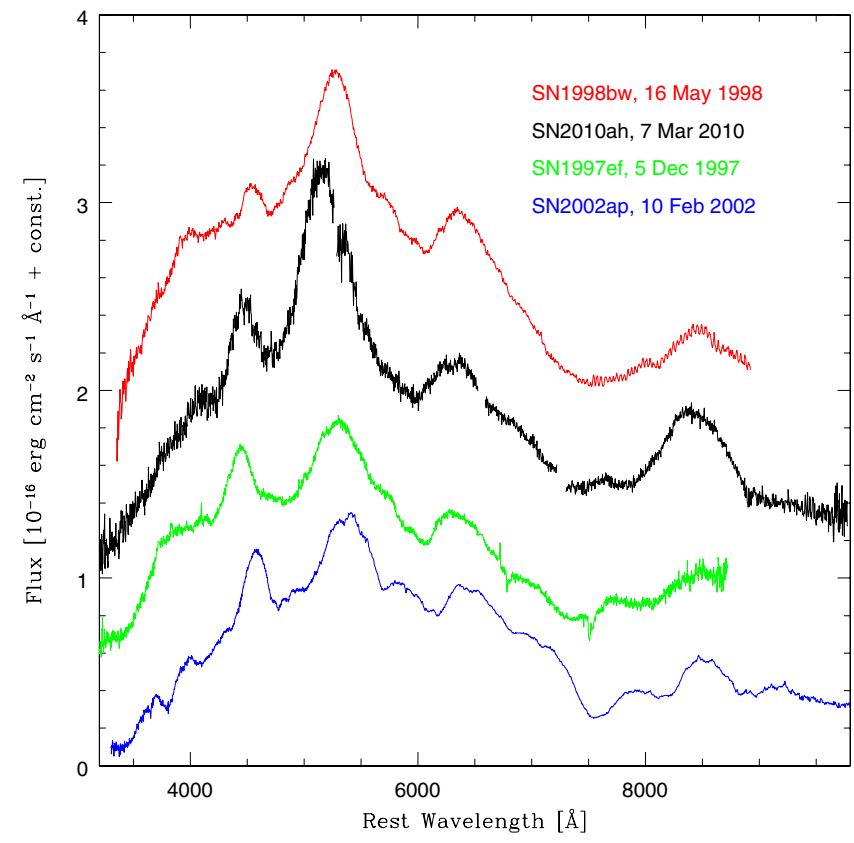

Figure 4. Keck (black) spectrum of PTF 10bzf obtained on 2010 March 7, about 5 days post $R$-band maximum. The spectrum is compared with that of SN 1997ef (green), SN 1998bw (red), and SN 2002ap (blue) at similar epochs. The SNe are ordered by peak luminosity. This ordering reveals a sequence also in line velocity and blending. The spectrum of SN 1998bw is the most affected by line broadening, as seen, for example, in the almost complete absence of the re-emission peak near $4500 \AA$ and by the complete blending of the O I 7773 and the Ca II IR triplet. PTF 10bzf is intermediate between SN 1998bw and the two non-GRB SNe 1997ef and 2002ap in both of these respects. Spectral data were retrieved from Mazzali et al. (2000) for SN 19997ef, Patat et al. (2001) for SN 1998bw, and Mazzali et al. (2002) for SN 2002ap. Main telluric lines have been removed from the PTF $10 \mathrm{bzf}$ spectrum.

(A color version of this figure is available in the online journal.)

\subsection{Swift Follow-up Observations}

A two-epoch observation of PTF 10bzf was performed as part of a Swift Target of Opportunity program. ${ }^{29}$ Swift/XRT did not detect any X-ray counterpart to PTF 10bzf (Kasliwal \& Cenko 2010). The corresponding upper limits are reported in Table 2, where we have converted the $0.3-10 \mathrm{keV}$ XRT count rates into fluxes assuming a photon index of $\Gamma=2$ and correcting for Galactic absorption $\left(N_{\mathrm{H}} \approx 10^{20} \mathrm{~cm}^{-2}\right)$.

During the first epoch, PTF 10bzf was detected by Swift/ UVOT (Kasliwal \& Cenko 2010) and was observed to fade in the subsequent observation. The measured magnitudes in Swift/ UVOT filters are reported in Table 2.

\subsection{Radio Follow-up Observations}

In the radio band, PTF 10bzf was followed up by the Allen Telescope Array (Welch et al. 2009), by the Combined Array for Research in Millimeter-wave Astronomy (CARMA; Carpenter 2010), and by the Westerbork Synthesis Radio Telescope (WSRT; Kamble et al. 2010). No radio source was detected by any of these telescopes (Table 2).

The Expanded Very Large Array ${ }^{30}$ (EVLA; Perley et al. 2009) provided the deepest upper limit of $33 \mu \mathrm{Jy}$ for a radio

\footnotetext{
29 "Unveiling New Classes of Transients with Palomar Transient Factory," PI: S. R. Kulkarni.

30 http://www.aoc.nrao.edu/evla/. The Very Large Array is operated by the National Radio Astronomy Observatory (NRAO), a facility of the National Science Foundation operated under cooperative agreement by Associated Universities, Inc.
}

counterpart associated with PTF 10bzf (Chomiuk \& Soderberg 2010, and Table 1).

Starting on 2010 November 27.49, we observed PTF 10bzf with the EVLA in its C configuration, through an EVLA exploratory program (PI: A. Corsi). ${ }^{31}$ We observed for 2 $\mathrm{hr}$, at a center frequency of $4.96 \mathrm{GHz}$, and with a total bandwidth of $256 \mathrm{MHz}$. No radio source was detected at the SN position; the corresponding upper limit is reported in Table 2. A compact source $(\mathrm{J} 1146+5356)$ near PTF 10bzf was observed every 6 minutes for accurate phase calibration, while 3C 286 was observed at the end of the run for the bandpass and flux density calibration. Data were reduced and imaged using the Astronomical Image Processing System (AIPS) software package.

We also reduced with the AIPS archival data that were taken on 2010 May 21.21 (PI: A. Soderberg), for a $1 \mathrm{hr}$ integration time at $6 \mathrm{GHz}$ (with a $1 \mathrm{GHz}$ bandwidth). We find no source at the position of PTF 10bzf and derive a $3 \sigma$ upper limit comparable to the one obtained by Chomiuk \& Soderberg (2010) during the first epoch (see Table 2).

\section{COMPARISON WITH SN 1998bw AND GRB SEARCH}

\subsection{Optical Emission}

Our photometric monitoring program missed the photometric maximum. However, by performing synthetic photometry on the Gemini spectrum taken on 2010 March 2 (about a week after the discovery), and the Keck spectrum taken on 2010 March 7 (about 12 days after the discovery), we derive $g-r-i$ magnitudes of PTF 10bzf at these epochs (see Table 2). As evident from Figure 2, PTF 10bzf was likely around maximum light during the Gemini observation. The $R$-band apparent magnitude of PTF 10bzf, as derived from the synthetic photometry on the Gemini spectrum, is $R=18.4 \pm 0.3$ (AB system or $R=$ $18.2 \pm 0.3$ in the Vega system). This gives us an approximate estimate of the $\mathrm{SN}$ absolute magnitude around $R$-band peak time of $M_{\mathrm{R}}=-18.3 \pm 0.3\left(\mathrm{AB}\right.$ system or $M_{\mathrm{R}}=-18.5 \pm 0.3$ in the Vega system).

In Figure 2 and Table 1 we summarize the properties of the spectroscopically confirmed broad-line Type Ic SNe associated with GRBs, of the broad-line Type Ic SNe 2003jd and 2002ap, as examples of "normal" broad-line Ics, and finally of the broadline Type Ic SN 2009bb (Pignata et al. 2011; Soderberg et al. 2010), as prototype of engine-driven SNe with no clear GRB association.

SNe 1998bw (Iwamoto et al. 1998), 2003dh (Mazzali et al. 2003), and 2003lw (Mazzali et al. 2007), all of which produced GRBs, were brighter and more energetic than other broadline Type Ic SNe for which a high-energy signal was either not detected (e.g., SNe 1997ef and SN 2002ap; see Mazzali et al. 2000, 2002) or was an XRF (SN 2006aj; Mazzali et al. 2006b). As evident from Table 1 and Figure 2, PTF 10bzf was more luminous than the broad-line Type Ic SN 2002ap (with a difference in $R$-band absolute magnitude at peak of $M_{\mathrm{R} ; 10 \mathrm{bzf}}-M_{\mathrm{R}, 2002 \mathrm{ap}}=-1.0 \pm 0.4$ ), comparable (within the errors) to the engine-driven SN 2009bb and to the broad-line Ic SN 2003jd at maximum $R$-band light, and less luminous than the GRB-associated SN $1998 \mathrm{bw}\left(M_{\mathrm{R}, 10 \mathrm{bzf}}-M_{\mathrm{R}, 1998 \mathrm{bw}}=0.9 \pm 0.3\right.$ at $R$-band maximum light).

Although the peak epoch is not well known, we can estimate the ${ }^{56} \mathrm{Ni}$ mass in PTF 10bzf by interpolating between the $R$-band

\footnotetext{
31 VLA/10C-227_-"Late-time follow-up of Type Ic SN PTF 10bzf."
} 
light curves of SN 2002ap (for which $M_{56 \mathrm{Ni}} \approx 0.09 M_{\odot}$; Mazzali et al. 2002) and SN 1998bw (for which $M_{56 \mathrm{Ni}} \approx 0.5 M_{\odot}$; Nakamura 1999; Nakamura et al. 2001). We find that for PTF 10bzf $M_{56 \mathrm{Ni}} \approx 0.20-0.25 M_{\odot}$. The ${ }^{56} \mathrm{Ni}$ mass can also be estimated using the empirical relation derived by Drout et al. (2010) from the properties of a large sample of Type Ic SNe (including broad-line and engine-driven $\mathrm{SNe}$ ):

$$
\log \left(M_{56 \mathrm{Ni}} / M_{\odot}\right) \simeq-0.41 \times M_{\mathrm{R}}-8.3,
$$

where $M_{56} \mathrm{Ni}$ is the mass of ${ }^{56} \mathrm{Ni}$ estimated using the formalism of Valenti et al. (2008; see also Arnett 1982) and $M_{\mathrm{R}}$ is the extinction-corrected peak magnitude in the $R$-band (see also Perets et al. 2010). For PTF 10bzf, using Equation (1), we get $M_{56} \mathrm{Ni} \approx 0.2 M_{\odot}$, compatible with what was obtained by interpolation. This is comparable to SN 2002aj, associated with XRF 060218 (Mazzali et al. 2006b), and significantly less than all other GRB-associated SNe (see Table 1).

Estimating the kinetic energy requires modeling. However, we can use line velocity as a proxy for it. In Figures 3 and 4 we show a spectroscopic sequence of broad-line Type Ic SNe, from 2002ap to 1998bw. The first (Figure 3) shows spectra near maximum light, the second (Figure 4) shows spectra obtained about 5 days after maximum. In addition, we show the only near-peak spectrum available of SN 1997ef, a broad-line SN without a GRB (Mazzali et al. 2000). We have ordered the SNe by peak luminosity. This ordering, however, reveals a sequence also in line velocity and blending. The spectra of SN 1998bw are the most affected by line broadening, as seen, for example, in the almost complete absence of the re-emission peak near $4500 \AA$ and by the complete blending of the O I 7773 and the Ca II IR triplet. PTF 10bzf is intermediate between SN 1998bw and the two non-GRB SNe 1997ef and 2002ap in both of these respects.

We have measured the velocity of the Si II $6355 \AA$ absorption, which traces reasonably closely the position of the photosphere, in the two available spectra of PTF 10bzf (see Figures 3 and 4). The results are shown in Figure 5, where they are compared to the photospheric velocity of a number of $\mathrm{Ib} / \mathrm{c}$ $\mathrm{SNe}$, with and without a GRB or an XRF. PTF 10bzf appears to be intermediate in velocity between the GRB-associated SNe (in red) and the non-GRB ones, both broad-lined (blue) and narrow-lined (green). Note that, typically, the measured Si II line velocity is somewhat larger than the photospheric velocity, so the photospheric velocity of PTF 10bzf is likely lower than the values shown in Figure 5. This makes PTF 10bzf more similar to non-GRB-associated SNe.

We can use this line velocity measurement, together with an estimate of the width of the light curve of PTF 10bzf, to derive an approximate estimate of the kinetic energy and ejecta mass for PTF 10bzf. As evident from Figure 2, the light curve of PTF 10bzf has to be stretched in time by a factor of $\approx 1.12$ to match the shape of the SN 1998bw light curve. Moreover, as evident from Figure 5, the velocity of PTF 10bzf may be a factor of $\approx 0.9$ smaller than SN 1998bw. Using the formula for the light curve width as a function of ejecta mass and kinetic energy, as delineated by Arnett (1982), we obtain for PTF 10bzf: $M=(7 \pm 2) M_{\odot}$ and $E_{\mathrm{K}}=(17 \pm 5) \times 10^{51} \mathrm{erg}$.

PTF 10bzf thus appears to have an $E_{\mathrm{K}} / M$ ratio of $\approx 2.5$. This is larger than non-GRB SNe such as 1997ef (which had $E_{\mathrm{K}} / M \approx 2$ ) or 2002ap (for which $E_{\mathrm{K}} / M \approx 1.25$ ), but smaller than SN 1998bw $\left(E_{\mathrm{K}} / M \approx 3\right)$. We thus conclude that PTF $10 \mathrm{bzf}$ probably lacks the mass and energy required to initiate a GRB.

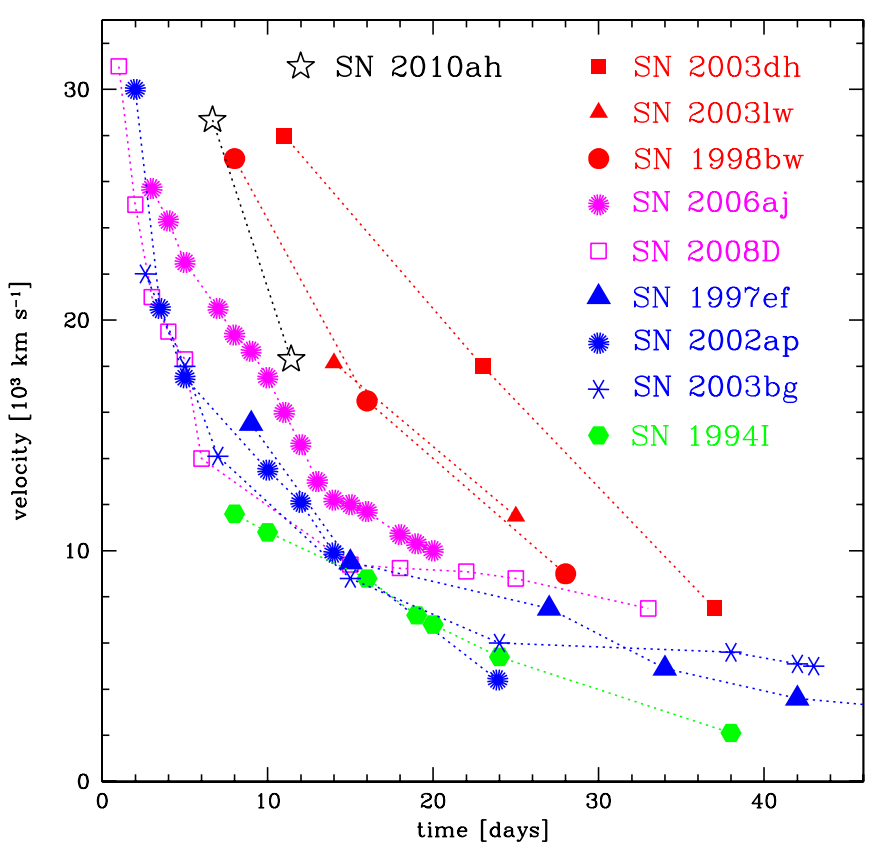

Figure 5. Measured Si II line velocity of PTF 10bzf (black stars) compared to photospheric velocities derived from spectroscopic modeling for a number of SNe Ib/c. Red symbols represent GRB-SNe (i.e., SN 1998bw, SN 2003dh, SN 2003lw; see Iwamoto et al. 1998; Mazzali et al. 2003, 2006b); magenta is used for XRF/X-ray transients-SNe (i.e., SN 2006aj and SN 2008D; Mazzali et al. 2006a; Pian et al. 2006; Modjaz et al. 2009); blue represents BL-SNe Type Ic (SN 1997ef and SN 2002ap; Mazzali et al. 2000, 2002) or IIb (SN 2003bg; Mazzali et al. 2009); finally green is used for the "normal" SN Ic 1994I (Sauer et al. 2006).

(A color version of this figure is available in the online journal.)

\subsection{X-Ray Upper Limit}

The Swift/XRT upper limit for PTF 10bzf at 8.5 days constrains any X-ray source associated with PTF 10bzf to have a flux $<1.3 \times 10^{-14} \mathrm{erg} \mathrm{s}^{-1} \mathrm{~cm}^{-2}$, which at the redshift of PTF 10bzf corresponds to an isotropic luminosity of $<7.4 \times$ $10^{40}$ erg s$^{-1}$.

The X-ray flux of GRB 980425 about 1 day after the burst was $\approx 3 \times 10^{-13} \mathrm{erg} \mathrm{s}^{-1} \mathrm{~cm}^{-2}$, and it declined at a rate $\propto t^{-0.2}$ (Nakamura 1999; Pian et al. 2000). Rescaling it at 8.5 days and taking into account the SN 1998bw distance, we obtain an $\mathrm{X}$-ray isotropic luminosity of $\approx 3 \times 10^{40} \mathrm{erg} \mathrm{s}^{-1}$, a factor of $\sim 2.4$ below our X-ray luminosity upper limit on PTF 10bzf.

For GRB 030329, an X-ray flux upper limit of $<2.6 \times$ $10^{-12} \mathrm{erg} \mathrm{s}^{-1} \mathrm{~cm}^{-2}$ was set at an epoch of about 8 days since the burst, using RXTE observations (Tiengo et al. 2003). From broadband afterglow modeling, the predicted $\mathrm{X}$-ray flux at 8 days was $\approx 4 \times 10^{-13} \mathrm{erg} \mathrm{s}^{-1} \mathrm{~cm}^{-2}$ (see Figure 4 in Tiengo et al. 2003), which at the redshift of GRB 030329 corresponds to an isotropic luminosity of $\approx 3 \times 10^{43} \mathrm{erg} \mathrm{s}^{-1}$, a factor of $\approx 400$ higher than our upper limit on PTF 10bzf.

GRB 031203 had an estimated X-ray flux of $\approx 10^{-13} \mathrm{erg} \mathrm{s}^{-1} \mathrm{~cm}^{-2}$ around 8 days (derived by extrapolating from the XMM-Newton observations; see Figure 1 in Watson et al. 2004). This corresponds to an isotropic X-ray luminosity of $\approx 3 \times 10^{42} \mathrm{erg} \mathrm{s}^{-1}$, a factor of $\approx 40$ higher than our X-ray luminosity upper limit on PTF 10bzf.

For GRB/XRF 060218, Swift/XRT measured a flux of $\approx 1.3 \times 10^{-13} \mathrm{erg} \mathrm{s}^{-1} \mathrm{~cm}^{-2}$ around 8 days since trigger (see the upper panel of Figure 2 in Campana et al. 2006). This 
gives an isotropic X-ray luminosity of $\approx 3 \times 10^{41} \mathrm{erg} \mathrm{s}^{-1}$, a factor of $\sim 4$ above our PTF 10bzf upper limit.

Finally, Swift/XRT observations set an upper limit of $\approx 3 \times$ $10^{-14} \mathrm{erg} \mathrm{s}^{-1} \mathrm{~cm}^{-2}$ on GRB $100316 \mathrm{D} X$-ray flux around 8 days since the burst (see Figure 6 in Starling et al. 2010), i.e., an isotropic luminosity upper limit of $\approx 2 \times 10^{41} \mathrm{erg} \mathrm{s}^{-1}$, a factor of $\sim 3$ greater than our upper limit on PTF 10bzf.

In conclusion, at $\approx 8$ days since trigger, all GRBs with a spectroscopically confirmed associated SN had an X-ray isotropic luminosity larger than our upper limit on PTF 10bzf, except for GRB 980425.

\subsection{Early-time ( $t \lesssim 20$ days) Radio Upper Limits}

The novelty of SN 1998bw was in the discovery of prompt radio emission just a few days after GRB 980425 (Kulkarni et al. 1998). The brightness temperature suggested that the radio photosphere moved relativistically $(\Gamma \geqslant 2)$, with a total energy of $\approx 10^{50} \mathrm{erg}(\mathrm{Li} \&$ Chevalier 1999), about two orders of magnitude less than the total kinetic energy of the explosion, which was estimated to be $\approx 3 \times 10^{52}$ erg (Iwamoto et al. 1998). None of these last features, together with the spatial and temporal association with a GRB, were ever observed for a nearby SN before SN 1998bw, thus suggesting the presence of a central engine powering this explosion.

SN 2003dh, associated with GRB 030329, showed an isotropic energy of $\approx 4 \times 10^{52} \mathrm{erg}$, which led to the classification of this event as an "hypernova" (Mazzali et al. 2003). The radio afterglow of GRB 030329 was $\gtrsim 100$ times more luminous than SN 1998bw (Berger et al. 2003a). Berger et al. (2003a) modeled such emission as due to the contribution of a second jet component, wider than the one generating the burst itself, and having an isotropic kinetic energy of $\approx 5.6 \times 10^{51} \mathrm{erg}$.

SN 20031w, associated with GRB 031203, had a total kinetic energy of $\approx 6 \times 10^{52} \mathrm{erg}$ (Mazzali et al. 2006b). The spectral peak luminosity in radio was $\approx 10^{29} \mathrm{erg} \mathrm{s}^{-1} \mathrm{~Hz}^{-1}$, about a factor of 100 less luminous than the radio afterglow of GRB 030329, but comparable to the radio peak luminosity of SN 1998bw. The kinetic energy in the afterglow component giving rise to the radio emission was estimated to be $\approx 1.7 \times 10^{49} \mathrm{erg}$ (Soderberg et al. 2004).

The total kinetic energy of SN 2006aj was $\approx 2 \times 10^{51} \mathrm{erg}$ (Mazzali et al. 2006a), smaller than the other GRB-associated SN. The radio afterglow of XRF 060218 peaked at about 5 days since the trigger, the peak luminosity being about a factor of 10 less bright than SN 1998bw at peak (Soderberg et al. 2006a). The kinetic energy and Lorentz factor around the time of the radio peak were estimated to be $\approx 2 \times 10^{48}$ erg and $\Gamma \approx 2.3$, respectively (Soderberg et al. 2006a).

SN 2010bh, the latest spectroscopically confirmed SN associated with a GRB, had a kinetic energy of $\approx 1.39 \times 10^{52} \mathrm{erg}$ (Cano et al. 2011). Radio upper limits at about 2 days since the burst constrain the radio spectral luminosity to be below $8 \times 10^{27} \mathrm{erg} \mathrm{s}^{-1} \mathrm{~Hz}^{-1}$, a factor of 2-10 lower than the radio afterglow luminosities observed for SN 1998bw, GRB 031203, and XRF 060218 on comparable timescales (Wieringa et al. 2010).

At a redshift of $z=0.0498$, the deepest early-time radio upper limit for PTF 10bzf was obtained by Chomiuk \& Soderberg (2010). This sets a limit on the spectral luminosity of $L_{5 \mathrm{GHz}}<$ $1.6 \times 10^{27} \mathrm{erg} \mathrm{s}^{-1} \mathrm{~Hz}^{-1}$, at about three weeks after the explosion. This is $\approx 20$ times lower than the radio luminosity observed on a similar timescale for the Type Ic SNe 1998bw and 2009bb (Soderberg et al. 2010), the only two SNe which showed clear

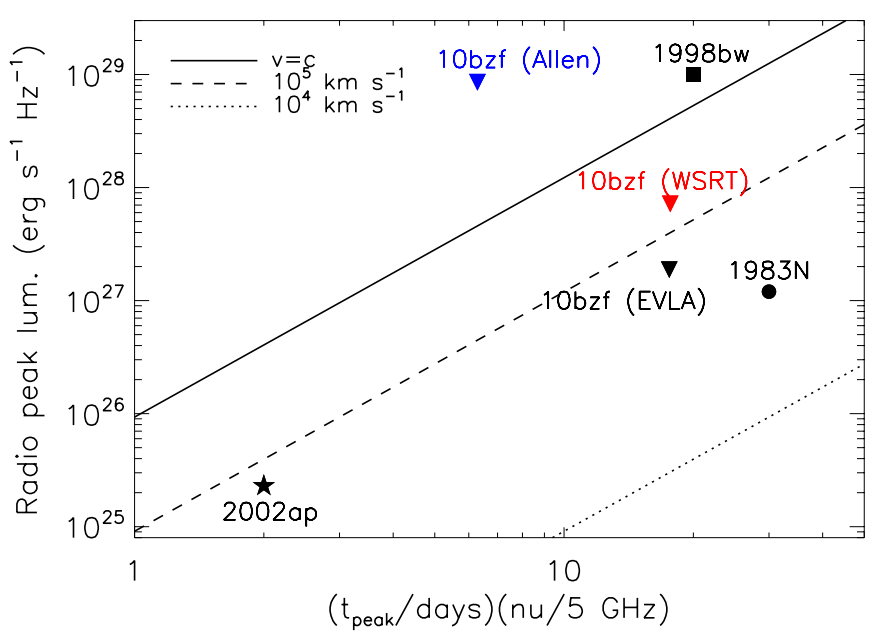

Figure 6. Constraints on approximate expansion velocity derived from the early-time Allen telescope (blue triangle), WSRT (red triangle), and EVLA (black triangle) radio upper limits on PTF 10bzf (see Table 2 and Equation (2)), compared with similar constraints obtained from the radio detections of SN 1998bw (black square), SN 1983N (black dot), and SN 2002ap (black star) (see Figure 2 in Berger et al. 2003b for comparison). As evident, SN 1998bw with $v_{\mathrm{r}} \sim c$ remains an exception. The Allen upper limit on PTF 10bzf around its peak time ( $\sim 10$ days since discovery, see Table 2$)$ does not exclude a relativistic expansion. However, $v \sim c$ is indeed excluded by the radio upper limits obtained about a week later by EVLA and WSRT. We stress that while the upper limits do not necessarily measure the peak of the spectrum at the time of the observation, typically time delays of few tens of days relative to the SN explosion reasonably sample the peak. However, if PTF 10bzf peaked at a few days after the explosion like SN 2002ap, the EVLA and WSRT upper limits would not be deep enough to rule out a relativistic expansion.

(A color version of this figure is available in the online journal.)

evidence for energetic and mildly relativistic outflows (Chomiuk \& Soderberg 2010). However, we note that this upper limit is comparable to the radio luminosity of GRB 060218 at a similar epoch (Soderberg et al. 2006a).

Using the early-time radio upper limit, we constrain the mean expansion speed $v_{\mathrm{r}}$ of the radio photosphere. Under the reasonable assumption that radio emission arises from a synchrotron spectrum with $v_{\mathrm{a}} \sim v_{\mathrm{p}}$ (where $v_{\mathrm{a}}$ is the selfabsorption frequency and $v_{\mathrm{p}}$ is the peak frequency), and under the hypothesis of equipartition, the peak time $t_{\mathrm{p}}$ and peak luminosity $L_{\mathrm{p}}$ of the radio emission directly measure the average expansion speed (Berger et al. 2003b; Chevalier 1998; Kulkarni et al. 1998; Scott \& Readhead 1977):

$$
\begin{aligned}
v_{\mathrm{r}} \sim 3.1 & \times 10^{4}\left(\frac{L_{\mathrm{p}}}{10^{26} \mathrm{erg} \mathrm{s}^{-1}}\right)^{17 / 36}\left(\frac{t_{\mathrm{p}}}{10 \text { days }}\right)^{-1} \\
& \times\left(\frac{v_{\mathrm{p}}}{5 \mathrm{GHz}}\right)^{-1} \mathrm{~km} \mathrm{~s}^{-1} .
\end{aligned}
$$

Figure 6 shows $L_{5 \mathrm{GHz}}$ at peak versus $t_{\mathrm{p}} v_{\mathrm{p}}$ for different values of $v_{\mathrm{r}}$, compared with the Allen telescope, WSRT, and EVLA upper limits on PTF 10bzf. Assuming that the radio emission peaked at $\approx 18$ days, the WSRT and EVLA observations constrain the expansion velocity to be less than $10^{5} \mathrm{~km} \mathrm{~s}^{-1}$.

Estimating the expansion velocities for non-detections has the caveat that the radio peak epoch is in reality unknown. However, as noted by Berger et al. (2003b), observations performed at $\sim 10-20$ days since explosion sample the radio peak time of Type Ic SNe reasonably well. For example, SN 1983N peaked in radio at $\sim 30$ days after explosion, while the radio light curve of SN 1998bw showed a double-peak profile, with the first peak around 10 days after explosion and the second around 30 days 
after explosion. This justifies our use of radio upper limits taken within $\approx 3$ weeks since discovery to constrain the expansion speed. We also note, however, that if PTF 10bzf peaked at a few days after the explosion like SN 2002ap, the EVLA and WSRT upper limits would not be deep enough to rule out a relativistic expansion.

\subsection{Late-time ( $t \gtrsim 20$ days) Radio Upper Limits}

In the context of the fireball model, the emission from an off-axis GRB is expected to be visible in the radio band long past the GRB explosion (at timescales of the order of $\sim 1 \mathrm{yr}$; see Levinson et al. 2002). In fact, at sufficiently late times, the relativistic fireball is expected to enter the sub-relativistic phase, during which the jet starts spreading, rapidly intersecting the viewer's line of sight as the ejecta approach spherical symmetry.

To model the late-time radio emission from an off-axis GRB during the non-relativistic phase, we use the analytical model of Waxman (2004b) for a fireball expanding in a wind medium (see, e.g., Levinson et al. 2002 for the constant ISM case). In this model the radio luminosity is approximated as (see also Perna \& Loeb 1998; Livio \& Waxman 2000; Paczynski 2001; Granot et al. 2002; Berger et al. 2003b; Soderberg et al. 2006b; van Eerten et al. 2010)

$$
\begin{aligned}
L_{\mathrm{r}} \sim 2.1 \times 10^{29}\left(\frac{\epsilon_{\mathrm{e}} \epsilon_{\mathrm{B}}}{0.01}\right)^{3 / 4} & \left(\frac{v}{10 \mathrm{GHz}}\right)^{-\frac{(p-1)}{2}}\left(\frac{t}{t_{\mathrm{NR}}}\right)^{-3 / 2} \\
& \times A_{*}^{9 / 4} E_{51}^{-1 / 2} \mathrm{erg} \mathrm{s}^{-1} \mathrm{~Hz}^{-1}
\end{aligned}
$$

where $E_{51}$ is the beaming-corrected ejecta energy, $A_{*}$ defines the circumstellar density in terms of the progenitor mass-loss rate $\dot{M}$ and wind velocity $v_{\mathrm{w}}$ such that $\dot{M} / 4 \pi v_{\mathrm{w}}=5 \times 10^{11} A_{*} \mathrm{~g} \mathrm{~cm}^{-1}$ (Waxman 2004b; Soderberg et al. 2006b), and

$$
t_{\mathrm{NR}} \sim 0.3\left(\frac{E_{51}}{A_{*}}\right) \mathrm{yr}
$$

is the time of the non-relativistic transition. Note that in the above formulation it is assumed that the fireball spreads sideways at approximately the speed of light. By imposing $t_{\mathrm{NR}} \lesssim t_{\mathrm{obs}}$ and $L_{\mathrm{r}} \gtrsim L_{\mathrm{obs}}$, we can use the EVLA observations of PTF 10bzf (see Table 2) to exclude the values of (beamingcorrected) energy and wind density that would give a radio luminosity above the upper limits of PTF 10bzf, at the times of our observations. The exclusion regions obtained in this way are plotted in yellow in Figure 7 . We can compare these constraints with the energy and density derived from the broadband afterglow modeling of GRB 980425, GRB 030329, and GRB 031203 (see Soderberg et al. 2006b; Waxman 2004a, and references therein), also plotted in Figure 7. From such a comparison we conclude that a GRB fireball possibly associated with PTF 10bzf could not have the same (beaming-corrected) energy and wind density as GRB 030329 and GRB 031203. In fact, if this was the case, such a fireball would have been in its non-relativistic (spherical) phase at the times of our radio observations (so that its radio emission would be observable by any off-axis observer), and its radio luminosity would be greater than our upper limits for PTF 10bzf.

We stress that our Figure 7 only constrains the portion of the parameter space where $t_{\mathrm{NR}} \lesssim t_{\text {obs }}$. This provides an estimate of the values of the fireball parameters ruled out by our observations, which does not depend critically on the adopted model. Constraining the portion of the parameter space where

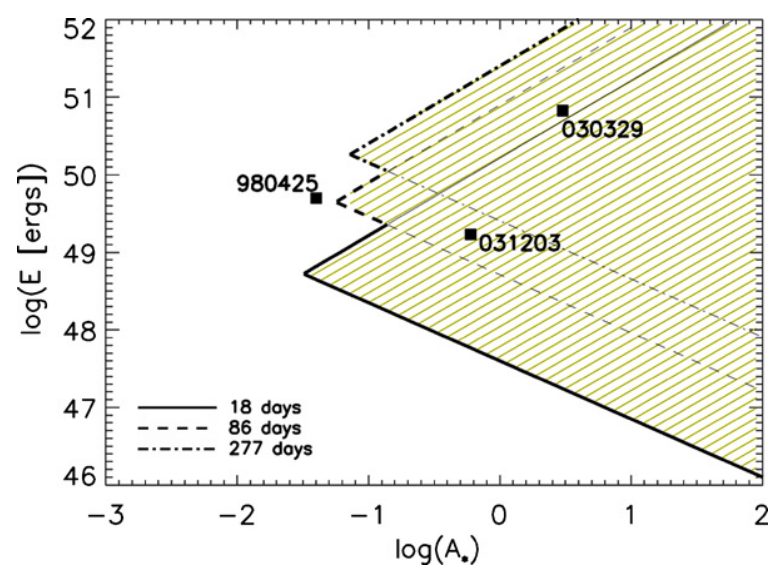

Figure 7. Off-axis emission from a GRB explosion associated with PTF 10bzf. The yellow shadowed regions mark the portion of the energy-density plane excluded by the first, second, and third epoch EVLA observations, respectively (see Table 2). As evident from the figure, late-time radio observations are fundamental to exclude the higher energy-higher density region of the energy-density plane. This region contains the most common values of energies and densities ( $E_{51} \sim 1-10$ and $A_{*} \sim 1$ ) observed for long GRBs. We also show for comparison some of the parameters estimated from broadband modeling of GRBs associated with SNe (980425, 030329, and 031203; see Soderberg et al. 2006b; Waxman 2004a, and references therein).

(A color version of this figure is available in the online journal.)

$t_{\mathrm{NR}} \gtrsim t_{\mathrm{obs}}$ requires detailed modeling. In fact, before $t_{\mathrm{NR}}$, the sideways expansion and the deceleration of the jet depend on the spatial distribution within the jet of the energy density and the Lorentz factor. These distributions are poorly constrained by current observations. And, for given distributions, an accurate calculation of jet expansion and deceleration must be carried out numerically.

\subsection{Search for $\gamma$-Rays}

We searched for a possible GRB in coincidence with PTF 10bzf using the interplanetary network (IPN) data. We searched for bursts with a localization error box including PTF 10bzf. Since the exact explosion epoch of PTF 10bzf is not known, we searched in a time window extending from 2010 February 12 to 2010 February 23 (the discovery day of PTF 10bzf). We conservatively extend such a time window to include one full week prior to our last non-detection of PTF 10bzf. Therefore, our time window starts $\approx 11$ days before the SN discovery (i.e., $\approx 18$ days before its $R$-band maximum light).

For comparison, we note that the engine-driven SN 2009bb reached its maximum light about two weeks after the estimated explosion date (Soderberg et al. 2010), evolving somewhat faster than SN 1998bw in the pre-peak phase (Pignata et al. 2011). As evident from Figure 2, our last upper limit on 2010 February $19(R>21.3)$ indicates that PTF 10bzf probably also evolved more rapidly than SN 1998 bw in its pre-peak phase. Using the $R$-band light curve as a proxy for $L(t)$, and assuming a pre-peak luminosity evolution $L(t) \propto\left(t-t_{0}\right)^{1.8}$ (where $t_{0}$ is the explosion time; see e.g., Pignata et al. 2011), $t_{0}$ should be in between $\approx 2010$ February 17.80 and $\approx 2010$ February 23.50 (i.e., the PTF discovery date) to account for the $\gtrsim 2.44$ mag drop observed between our last non-detection of PTF 10bzf and its discovery. However, we conservatively extend our time interval for the GRB search to 2010 February 12, one week prior to our last non-detection (on 2010 February 19.443). 
Between 2010 February 12 and 2010 February 23, a total of 14 confirmed bursts were detected by the spacecraft of the IPN ${ }^{32}$ (Mars Odyssey, Konus-Wind, RHESSI, INTEGRAL (SPI-ACS), Swift-BAT, MESSENGER, Suzaku, AGILE, and Fermi (GBM)). Here confirmed means that they were observed by more than one detector on one or more spacecraft and could be localized at least coarsely.

The localization accuracies of these 14 bursts varied widely, but none of them was found to be consistent with the position of PTF 10bzf. One burst was observed by the Fermi GBM alone (error circles with $1 \sigma$ statistical-only error radii of $10.5 \mathrm{deg}$ ), while another five were observed by the GBM and other spacecraft. Four events were observed by a distant IPN spacecraft and could be triangulated to annuli or error boxes with dimensions as small as $\sim 10^{\prime}$. Five GRBs were observed within the coded field of view of the Swift-BAT ( $3^{\prime}$ initial localization accuracy), and in some cases by other IPN spacecraft as well.

PTF 10bzf is not spatially associated with any of these GRBs. The total area of the localizations of the 14 confirmed bursts, containing the $3 \sigma$ error regions, was $\approx 1.3 \mathrm{sr}$. Therefore, there is only $\approx 10 \%$ chance coincidence between these bursts and PTF 10bzf.

Next, based on our GRB sample, we can put a limit on the fluence of any GRB associated with PTF 10bzf. We considered three distinct sets of events: IPN bursts, Fermi GBM-only bursts, and Swift-BAT-only bursts. The IPN is sensitive to bursts with fluences down to about $6 \times 10^{-7} \mathrm{erg} \mathrm{cm}^{-2}$ (50\% efficiency-see Hurley et al. 2010) and observes the entire sky with a temporal duty cycle close to $100 \%$. The Fermi GBM detects bursts down to a $8-1000 \mathrm{keV}$ fluence of about $4 \times 10^{-8} \mathrm{erg} \mathrm{cm}^{-2}$ and observes the entire unocculted sky $(\approx 8.8 \mathrm{sr}$ ) with a temporal duty cycle of more than $80 \%$. The weakest burst observed by the BAT had a $15-150 \mathrm{keV}$ fluence of $6 \times 10^{-9} \mathrm{erg} \mathrm{cm}^{-2}$, and the BAT observes a field of view of about 2 sr with a temporal duty cycle of about $90 \%$.

If the SN produced a burst below the IPN threshold, and above the Fermi one, it is possible that both Swift and Fermi did not detect it: considering their spatial and temporal coverages, the non-detection probabilities are about 0.86 and 0.44 , respectively. Finally, if the SN produced a burst below the Fermi threshold, but above the Swift one, the non-detection probability is about 0.86 . We note that a burst with an isotropic energy release comparable to that of the sub-energetic GRB 980425, $E_{\text {iso }} \approx 6 \times 10^{47} \mathrm{erg}$, placed at the distance of PTF 10bzf, would be observed with a fluence of $\approx 10^{-7} \mathrm{erg} \mathrm{cm}^{-2}$, which is below the IPN threshold, but above the Fermi GBM one.

\section{CONCLUSION}

We presented the discovery of PTF 10bzf, a broad-line Type Ic SN detected by PTF. We obtained multi-wavelength followup observations of this $\mathrm{SN}$, compared its properties with those of other SNe associated with GRBs, and put limits on any associated GRB using the IPN sample.

While PTF 10bzf shows some spectral similarities with SN 1998bw, its $R$-band and radio luminosities are much lower, with no clear evidence for a relativistic expansion speed. The spectral properties of PTF 10bzf suggest that this SN is intermediate, in terms of explosion kinetic energy, between non-GRB-associated SNe like 2002ap or 1997ef and GRBassociated SNe like 1998bw. A search for $\gamma$-rays using the IPN sample gives no GRB with a position consistent with this

\footnotetext{
32 http://www.ssl.berkeley.edu/ipn3/
}

$\mathrm{SN}$, in a time window extending to a full week prior to our last non-detection of the source. We thus conclude that PTF 10bzf probably lacks the mass and energy required to initiate a GRB.

Despite the fact that PTF 10bzf does not show evidence for being associated with a nearby GRB, the discovery and followup of broad-line Type Ic SNe remains a fundamental tool to investigate the GRB-SN connection. Broad-line Type Ic SNe are rare, and they are the only type of SNe which have confirmed associations with GRBs. Therefore, it is crucial to study them and determine how the GRB-associated SNe differ from the other broad-line Type Ic SNe. PTF is a project able to discover and classify about 10 broad-line Type Ic SNe per year, thus allowing the construction of a large sample of $\mathrm{SNe}$ which are unique broad-line Ic.

The search for $\mathrm{SNe}$ associated with nearby (non- $\gamma$-ray triggered) GRBs is particularly relevant also in the light of multi-messenger astronomy. In an era in which ground-based gravitational wave detectors such as $\mathrm{LIGO}^{33}$ and $\mathrm{Virgo}^{34}$ are approaching their advanced configurations, nearby GRBs represent promising candidates for the detection of gravity waves (e.g., Kobayashi \& Mészáros 2003; Kokkotas 2004; Woosley \& Bloom 2006; Piro \& Pfahl 2007; Corsi \& Mészáros 2009; Ott 2009, and references therein). The simultaneous operation of facilities such as PTF and LIGO may open, in the forthcoming years, a unique opportunity for this kind of multi-messenger searches (e.g., Bloom et al. 2009; Smith et al. 2009; Stamatikos et al. 2009; Shawhan 2010, and references therein).

PTF is a collaboration of Caltech, LCOGT, the Weizmann Institute, LBNL, Oxford, Columbia, IPAC, and Berkeley.

The National Energy Research Scientific Computing Center, which is supported by the Office of Science of the U.S. Department of Energy under Contract No. DE-AC02-05CH11231, provided staff, computational resources, and data storage for this project.

E.O.O. is supported by an Einstein Fellowship and NASA grants. D.P. is supported by an Einstein fellowship.

The Weizmann Institute PTF partnership is supported in part by grants from the Israeli Science Foundation (ISF) to A.G. Joint work by the Weizmann and Caltech groups is supported by a grant from the Binational Science Foundation (BSF) to A.G. and S.R.K. A.G. acknowledges further support from an EU/FP7 Marie Curie IRG fellowship and a research grant from the Peter and Patricia Gruber Awards.

The National Radio Astronomy Observatory is a facility of the National Science Foundation operated under cooperative agreement by Associated Universities, Inc.

The Gemini Observatory is operated by the Association of Universities for Research in Astronomy, Inc., under a cooperative agreement with the NSF on behalf of the Gemini partnership: the National Science Foundation (United States), the Science and Technology Facilities Council (United Kingdom), the National Research Council (Canada), CONICYT (Chile), the Australian Research Council (Australia), Ministério da Ciência e Tecnologia (Brazil), and Ministerio de Ciencia, Tecnología e Innovación Productiva (Argentina).

The W. M. Keck Observatory is operated as a scientific partnership among the California Institute of Technology, the University of California and the National Aeronautics and Space Administration. The Observatory was made possible by the generous financial support of the W. M. Keck Foundation.

\footnotetext{
33 www.ligo.org

34 www.virgo.infn.it
} 
LAIWO, a wide-angle camera operating on the $1 \mathrm{~m}$ telescope at the Wise Observatory, Israel, was built at the Max Planck Institute for Astronomy (MPIA) in Heidelberg, Germany, with financial support from the MPIA, and grants from the German Israeli Science Foundation for Research and Development, and from the Israel Science Foundation.

LIGO was constructed by the California Institute of Technology and Massachusetts Institute of Technology with funding from the National Science Foundation.

We are grateful to IPN collaborators S. Golenetskii, R. Aptekar, E. Mazets, D. Frederiks, and T. Cline for the Konus data; to M. Briggs and C. Meegan for the Fermi data; to T. Takahashi, Y. Terada, M. Tashiro, Y. Fukazawa, T. Murakami, M. Ohno, and K. Makishima for the Suzaku data; to S. Barthelmy, N. Gehrels, H. Krimm, and D. Palmer for the Swift data; to D. Golovin, A. Kozyrev, M. Litvak, A. Sanin, C. Fellows, K. Harshman, and R. Starr for the Odyssey data; and to $\mathrm{A}$. von Kienlin and $\mathrm{X}$. Zhang for the INTEGRAL data. K.H. acknowledges support from the following NASA sources: NNX09AV61G (Suzaku), NNX10AI23G (Swift), NNX10AU34G (Fermi), and NNX07AR71G (MESSENGER).

S.B.C. acknowledges generous financial assistance from Gary \& Cynthia Bengier, the Richard \& Rhoda Goldman Fund, NASA/Swift grants NNX10AI21G and GO-7100028, the TABASGO Foundation, and NSF grant AST-0908886.

A.C. and S.R.K. acknowledge partial support from NASA/ Swift Guest Investigator Program Cycle 7 (NNH10ZDA001N).

\section{REFERENCES}

Abdo, A. A., Ackermann, M., Ajello, M., et al. 2009a, Nature, 462, 331 Abdo, A. A., Ackermann, M., Ajello, M., et al. 2009b, ApJ, 706, L138 Abdo, A. A., Ackermann, M., Arimoto, M., et al. 2009c, Science, 323, 1688 Arcavi, I., Gal-Yam, A., Kasliwal, M. M., et al. 2010, ApJ, 721, 777 Arnett, W. D. 1982, ApJ, 253, 785

Balberg, S., \& Loeb, A. 2011, MNRAS, 414, 1715

Berger, E., Kulkarni, S. R., Pooley, G., et al. 2003a, Nature, 426, 154 Berger, E., Kulkarni, S. R., Pooley, G., et al. 2003b, ApJ, 599, 408 Bersier, D., Rhoads, J., Fruchter, A., et al. 2004, GRB Coordinates Network, 2544, 1

Bloom, J. S., Frail, D. A., \& Kulkarni, S. R. 2003, ApJ, 594, 674

Bloom, J. S., Holz, D. E., Hughes, S. A., et al. 2009, arXiv:0902.1527

Bucciantini, N., Quataert, E., Arons, J., Metzger, B. D., \& Thompson, T. A 2008, MNRAS, 383, L25

Bufano, F., Benetti, S., Cappellaro, E., et al. 2010, CBET, 2227, 1

Campana, S., Mangano, V., Blustin, A. J., et al. 2006, Nature, 442, 1008

Cano, Z., Bersier, D., Guidorzi, C., et al. 2011, arXiv:1104.5141

Carpenter, J. M. 2010, ATel, 2473, 1

Chevalier, R. A. 1976, ApJ, 207, 872

Chevalier, R. A. 1992, ApJ, 394, 599

Chevalier, R. A. 1998, ApJ, 499, 810

Chevalier, R. A., \& Fransson, C. 2008, ApJ, 683, L135

Chomiuk, L., \& Soderberg, A. 2010, ATel, 2483, 1

Chornock, R., Berger, E., Levesque, E. M., et al. 2010, arXiv:1004.2262

Cobb, B. E., Bailyn, C. D., van Dokkum, P. G., Buxton, M. M., \& Bloom, J. S. 2004, ApJ, 608, L93

Corsi, A., Guetta, D., \& Piro, L. 2010, ApJ, 720, 1008

Corsi, A., \& Mészáros, P. 2009, ApJ, 702, 1171

Della Valle, M., Chincarini, G., Panagia, N., et al. 2006, Nature, 444, 1050

Deng, J., Tominaga, N., Mazzali, P. A., Maeda, K., \& Nomoto, K. 2005, ApJ, 624,898

Drout, M. R., Soderberg, A. M., Gal-Yam, A., et al. 2010, arXiv:1011.4959

Ferrero, P., Kann, D. A., Zeh, A., et al. 2006, A\&A, 457, 857

Filippenko, A. V. 1997, ARA\&A, 35, 309

Foley, R. J., Papenkova, M. S., Swift, B. J., et al. 2003, PASP, 115, 1220

Frail, D. A., Kulkarni, S. R., Sari, R., et al. 2001, ApJ, 562, L55

Fruchter, A. S., Levan, A. J., Strolger, L., et al. 2006, Nature, 441, 463

Fynbo, J. P. U., Jakobsson, P., Möller, P., et al. 2003, A\&A, 406, L63

Fynbo, J. P. U., Watson, D., Thöne, C. C., et al. 2006, Nature, 444, 1047

Gal-Yam, A., Fox, D. B., Price, P. A., et al. 2006a, Nature, 444, 1053

Gal-Yam, A., Ofek, E. O., Poznanski, D., et al. 2006b, ApJ, 639, 331
Gal-Yam, A., Ofek, E. O., \& Shemmer, O. 2002, MNRAS, 332, L73

Gal-Yam, A., Maoz, D., Guhathakurta, P., \& Filippenko, A. V. 2008, ApJ, 680, 550

Gal-Yam, A., Moon, D.-S., Fox, D. B., et al. 2004, ApJ, 609, L59

Galama, T. J., Vreeswijk, P. M., van Paradijs, J., et al. 1998, Nature, 395, 670

Garnavich, P., Matheson, T., Olszewski, E. W., et al. 2003, IUAU Circ., 8114, 1

Gezari, S., Dessart, L., Basa, S., et al. 2008, ApJ, 683, L131

Granot, J., Panaitescu, A., Kumar, P., \& Woosley, S. E. 2002, ApJ, 570, L61

Grassberg, E. K., Imshennik, V. S., \& Nadyozhin, D. K. 1971, Ap\&SS, 10, 28

Guetta, D., \& Della Valle, M. 2007, ApJ, 657, L73

Hjorth, J., Sollerman, J., Møller, P., et al. 2003, Nature, 423, 847

Hook, I. M., Jørgensen, I., Allington-Smith, J. R., et al. 2004, PASP, 116, 425

Hurley, K., Cline, T., Mitrofanov, I., et al. 2010, in The Shocking Universe Gamma Ray Bursts and High Energy Shock Phenomena, ed. G. Chincarini,

P. D’Avanzo, R. Margutti, \& R. Salvaterra, Sociatá Italiana di Fisica Conf. Proc., Vol. 102, 529

Iwamoto, K., Mazzali, P. A., Nomoto, K., et al. 1998, Nature, 395, 672

Jordi, K., Grebel, E. K., \& Ammon, K. 2006, A\&A, 460, 339

Kamble, A. P., van der Horst, A. J., Paragi, Z., et al. 2010, ATel, 2479, 1

Kasliwal, M. M., \& Cenko, S. B. 2010, ATel, 2471, 1

Katz, B., Budnik, R., \& Waxman, E. 2010, ApJ, 716, 781

Katz, B., Sapir, N., \& Waxman, E. 2011, arXiv:1106.1898

Kawabata, K. S., Deng, J., Wang, L., et al. 2003, ApJ, 593, L19

Kobayashi, S., \& Mészáros, P. 2003, ApJ, 589, 861

Kokkotas, K. D. 2004, Class. Quantum Grav., 21, 501

Kulkarni, S. R., Frail, D. A., Wieringa, M. H., et al. 1998, Nature, 395, 663

Law, N. M., Kulkarni, S. R., Dekany, R. G., et al. 2009, PASP, 121, 1395

Levinson, A., Ofek, E. O., Waxman, E., Gal-Yam, A., et al. 2002, ApJ, 576, 923

Li, Z., \& Chevalier, R. A. 1999, ApJ, 526, 716

Liang, E., Zhang, B., Virgili, F., \& Dai, Z. G. 2007, ApJ, 662, 1111

Liang, E.-W., Racusin, J. L., Zhang, B., et al. 2008, ApJ, 675, 528

Liang, E.-W., Yi, S.-X., Zhang, J., et al. 2010, ApJ, 725, 2209

Lithwick, Y., \& Sari, R. 2001, ApJ, 555, 540

Livio, M., \& Waxman, E. 2000, ApJ, 538, 187

MacFadyen, A. I., \& Woosley, S. E. 1999a, ApJ, 524, 262

MacFadyen, A. I., \& Woosley, S. E. 1999b, ApJ, 524, 262

Malesani, D., Tagliaferri, G., Chincarini, G., et al. 2004, ApJ, 609, L5

Matheson, T., Garnavich, P. M., Stanek, K. Z., et al. 2003, ApJ, 599, 394

Mazzali, P. A., Deng, J., Hamuy, M., \& Nomoto, K. 2009, ApJ, 703, 1624

Mazzali, P. A., Deng, J., Maeda, K., et al. 2002, ApJ, 572, L61

Mazzali, P. A., Deng, J., Nomoto, K., et al. 2006a, Nature, 442, 1018

Mazzali, P. A., Deng, J., Pian, E., et al. 2006b, ApJ, 645, 1323

Mazzali, P. A., Deng, J., Tominaga, N., et al. 2003, ApJ, 599, L95

Mazzali, P. A., Iwamoto, K., \& Nomoto, K. 2000, ApJ, 545, 407

Mazzali, P. A., Kawabata, K. S., Maeda, K., et al. 2005, Science, 308, 1284

Mazzali, P. A., Kawabata, K. S., Maeda, K., et al. 2007, ApJ, 670, 592

Mészáros, P. 2006, Rep. Prog. Phys., 69, 2259

Metzger, B. D., Giannios, D., Thompson, T. A., Bucciantini, N., \& Quataert, E.

2011, MNRAS, 413, 2031

Modjaz, M., Kewley, L., Kirshner, R. P., et al. 2008, AJ, 135, 1136

Modjaz, M., Li, W., Butler, N., et al. 2009, ApJ, 702, 226

Modjaz, M., Stanek, K. Z., Garnavich, P. M., et al. 2006, ApJ, 645, L21

Nagakura, H., Ito, H., Kiuchi, K., \& Yamada, S. 2011, ApJ, 731, 80

Nakamura, T. 1999, ApJ, 522, L101

Nakamura, T., Mazzali, P. A., Nomoto, K., \& Iwamoto, K. 2001, ApJ, 550, 991

Nakar, E., \& Sari, R. 2010, ApJ, 725, 904

Norris, J. P. 2002, ApJ, 579, 386

Ofek, E. O., Cenko, S. B., Gal-Yam, A., et al. 2007, ApJ, 662, 1129

Ofek, E. O., Kasliwal, M. M., Gal-Yam, A., et al. 2010a, ATel, 2470, 1

Ofek, E. O., Rabinak, I., Neill, J. D., et al. 2010b, ApJ, 724, 1396

Ott, C. D. 2009, Class. Quantum Grav., 26, 204015

Paczynski, B. 2001, Acta Astron., 51, 1

Patat, F., Cappellaro, E., Danziger, J., et al. 2001, ApJ, 555, 900

Perets, H. B., Gal-Yam, A., Mazzali, P. A., et al. 2010, Nature, 465, 322

Perley, R., Napier, P., Jackson, J., et al. 2009, IEEE Proc., 97, 1448

Perna, R., \& Loeb, A. 1998, ApJ, 509, L85

Pian, E., Amati, L., Antonelli, L. A., et al. 2000, ApJ, 536, 778

Pian, E., Mazzali, P. A., Masetti, N., et al. 2006, Nature, 442, 1011

Pignata, G., Stritzinger, M., Soderberg, A., et al. 2011, ApJ, 728, 14

Piran, T. 1999, Phys. Rep, 314, 575

Piran, T. 2004, Rev. Mod. Phys., 76, 1143

Piro, A. L., \& Pfahl, E. 2007, ApJ, 658, 1173

Podsiadlowski, P., Mazzali, P. A., Nomoto, K., Lazzati, D., \& Cappellaro, E. 2004, ApJ, 607, L17

Poznanski, D., Gal-Yam, A., Maoz, D., et al. 2002, PASP, 114, 833

Proga, D., \& Begelman, M. C. 2003, ApJ, 592, 767

Rabinak, I., \& Waxman, E. 2011, ApJ, 728, 63 
Racusin, J. L., Liang, E. W., Burrows, D. N., et al. 2009, ApJ, 698, 43 Rau, A., Kulkarni, S. R., Law, N. M., et al. 2009, PASP, 121, 1334 Rhoads, J. E. 2003, ApJ, 591, 1097

Sauer, D. N., Mazzali, P. A., Deng, J., et al. 2006, MNRAS, 369, 1939

Schawinski, K., Justham, S., Wolf, C., et al. 2008, Science, 321, 223

Schlegel, D. J., Finkbeiner, D. P., \& Davis, M. 1998, ApJ, 500, 525

Scott, M. A., \& Readhead, A. C. S. 1977, MNRAS, 180, 539

Shawhan, P. S., LIGO Scientific Collaboration, \& Virgo Collaboration 2010, BAAS, 42, 230

Smith, J., LIGO Scientific Collaboration, \& Virgo Collaboration 2009, BAAS, 41,443

Soderberg, A. M., Berger, E., Page, K. L., et al. 2008, Nature, 453, 469

Soderberg, A. M., Chakraborti, S., Pignata, G., et al. 2010, Nature, 463, 513

Soderberg, A. M., Kulkarni, S. R., Berger, E., et al. 2004, Nature, 430, 648

Soderberg, A. M., Kulkarni, S. R., Nakar, E., et al. 2006a, Nature, 442, 1014

Soderberg, A. M., Nakar, E., Berger, E., \& Kulkarni, S. R. 2006b, ApJ, 638, 930

Sollerman, J., Jaunsen, A. O., Fynbo, J. P. U., et al. 2006, A\&A, 454, 503

Stamatikos, M., Gehrels, N., Halzen, F., Mészáros, P., \& Roming, P.W.A. 2009 arXiv:0902.3022

Stanek, K. Z., Matheson, T., Garnavich, P. M., et al. 2003, ApJ, 591, L17
Starling, R. L. C., Wiersema, K., Levan, A. J., et al. 2011, MNRAS, 411, 2792

Thompson, T. A., Chang, P., \& Quataert, E. 2004, ApJ, 611, 380

Tiengo, A., Mereghetti, S., Ghisellini, G., et al. 2003, A\&A, 409, 983

Toma, K., Ioka, K., Sakamoto, T., \& Nakamura, T. 2007, ApJ, 659, 1420

Usov, V. V. 1992, Nature, 357, 472

Valenti, S., Benetti, S., Cappellaro, E., et al. 2008, MNRAS, 383, 1485

van Eerten, H., Zhang, W., \& MacFadyen, A. 2010, ApJ, 722, 235

Virgili, F. J., Liang, E., \& Zhang, B. 2009, MNRAS, 392, 91

Watson, D., Hjorth, J., Levan, A., et al. 2004, ApJ, 605, L101

Waxman, E. 2004a, ApJ, 605, L97

Waxman, E. 2004b, ApJ, 602, 886

Waxman, E., Mészáros, P., \& Campana, S. 2007, ApJ, 667, 351

Welch, J., Backer, D., Blitz, L., et al. 2009, IEEE Proc, 97, 1438

Wieringa, M., Soderberg, A., \& Edwards, P. 2010, GCN Circ., 10533, 1

Wiersema, K., D’Avanzo, P., Levan, A. J., et al. 2010, GCN Circ., 10525, 1

Woosley, S. E. 1993, ApJ, 405, 273

Woosley, S. E., \& Bloom, J. S. 2006, ARA\&A, 44, 507

Woosley, S. E., \& Heger, A. 2006, ApJ, 637, 914

Woosley, S. E., \& Zhang, W. 2007, Phil. Trans. R. Soc. A, 365, 1129

York, D. G., Adelman, J., Anderson, J. E. Jr., et al. 2000, AJ, 120, 1579

Zhang, B., Kobayashi, S., \& Mészáros, P. 2003, ApJ, 595, 950 